\title{
Cognitive modeling with spreadsheets
}

\author{
SIEGFRIED MACHO \\ University of Fribourg, Fribourg, Switzerland
}

\begin{abstract}
Spreadsheet implementations of two different types of cognitive models-a neural network model and a statisticalmodel-are presented. The two examples illustrate how to employ the facilities of spreadsheets, the spreadsheet data structure, array functions, the built-in function library, and the integrated optimizer, for building cognitive models. The two presented models are new extensions of existing models. They are used for simulating data from experiments illustrating that the extended versions are able to explain experimental results that could not be simulated by the original models. The whole simulation study demonstrates that spreadsheets are a handy tool, especially for researchers without programming knowledge who want to build cognitive models and for instructors teaching cognitive modeling.
\end{abstract}

Developing and testing models of cognition usually requires programming skills or special purpose software. Building an efficient and flexible model using conventional programming languages is difficult for many people. Special purpose software has at least four disadvantages: (1) It requires learning (or relearning) the use of the program; (2) it can be inflexible, making it difficult to implement extensions of existing models; (3) the details of the implementation may be hidden, making it unsuitable for teaching purposes; and (4) it may be costly.

By contrast, spreadsheets are an ideal means for developing and testing models of cognition, as well as for teaching cognitive models. They are well understood, flexible, transparent, and readily available.

A spreadsheet (e.g., Excel) is a data structure that carries a variety of data objects, including text, numbers, graphical objects, and computational formulas. In addition, the spreadsheet program provides a function library and an optimization module. Thus, a spreadsheet is a medium for integrating different computational structures (i.e., computational formulas and procedures), as well as noncomputational structures (e.g., graphical objects). Linking computational formulas enables one to implement complex computational models without specific programming skills.

This illustration extends two existing cognitive models: (1) the configural model of Pearce (1994), and (2) a multinomial processing tree signal detection model. These represent two classes of models: The configural model of Pearce is a neural network model for simulating qualitatively associative learning processes, whereas the multinomial processing tree signal detection model is a statis-

The author thanks Odilo W. Huber, Oswald Huber, Jonathan Vaughan, and two anonymous reviewers for their comments on an earlier version of this article and thanks Andrew Yonelinas for making available his data. Correspondence should be addressed to S. Macho, University of Fribourg, Rue Faucigny 2, CH 1700 Fribourg, Switzerland (e-mail: siegfried. macho@unifr.ch). tical model for predicting frequency distributions over response categories in memory recognition experiments. The extended versions of these two models relax certain restrictions so that they are able to simulate experimental data that could not be fitted by the original models. The models were implemented using Microsoft Excel 97 (for Windows) and tested for the versions Excel 2000 (for Windows), and Excel 98 and 2001 (both for Macintosh). The implementation did not exploit macros or Visual Basic, so the principles should transfer to other platforms, such as Quattro Pro (Corel Corporation; www.corel.com) or Lotus 1-2-3 (IBM; www.ibm.com). The workbooks that implement the models can be retrieved from http://www.unifr. ch/psycho/general/macho/cognitive.htm.

\section{THE CONFIGURAL MODEL OF PEARCE (CMP)}

The CMP is an associative learning model for simulating contingency and causal learning (Baker, ValléeTourangeau, \& Murphy, 2000; Pearce, 1987, 1994; ValléeTourangeau, Murphy, \& Baker, 1998; Vallée-Tourangeau, Murphy, Drew, \& Baker, 1998; Van Overwalle \& Rooy, 1998). In the following, the model will be implemented as a neural network.

\section{Description of the Model}

Figure 1 shows the architecture of the network that comprises three layers: an input layer, a configural layer, and an output layer.

The input layer represents the input pattern of a given learning trial. The input pattern is a $0 / 1$ vector, with 1 indicating the presence of a cue and 0 representing the absence of a cue. In Figure 1, the input layer represents the input

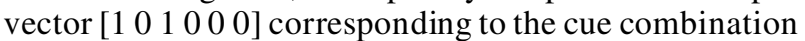
$A C$. The gray colored circles indicate active input units.

Each configural unit in the configural layer contains a configural pattern/vector that corresponds to a possible input pattern. For example, the first configural unit in Figure 1 contains the configural vector $\left[\begin{array}{llllll}1 & 0 & 0 & 0 & 0 & 0\end{array}\right]$, repre- 


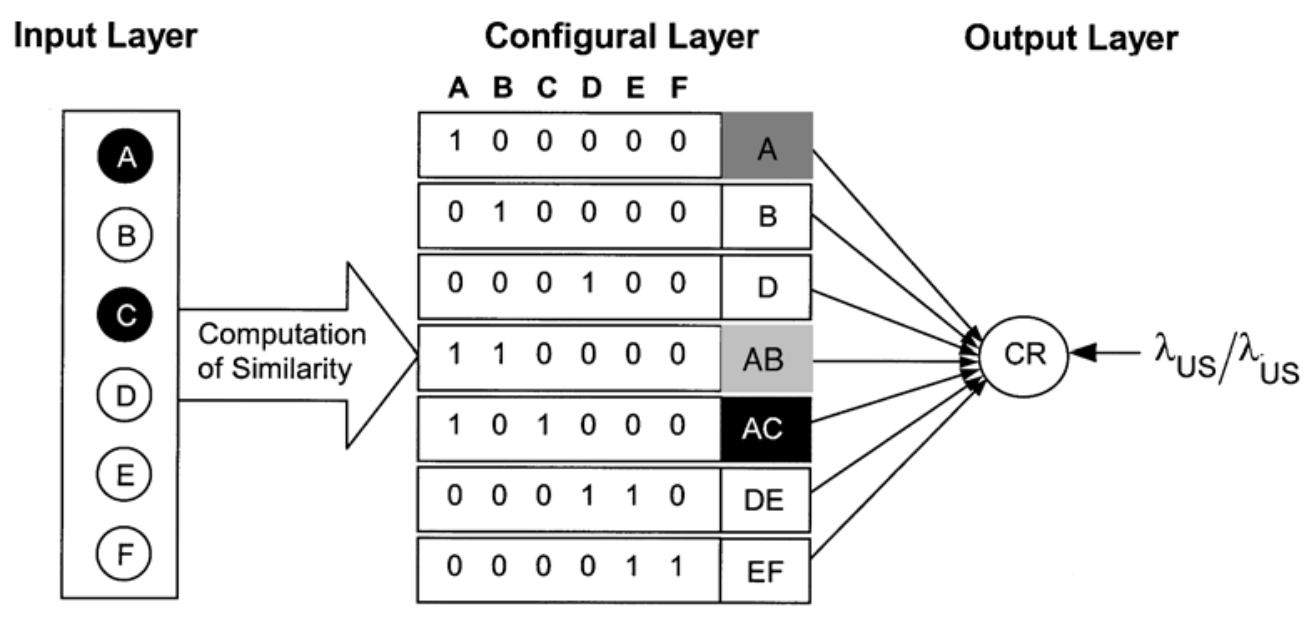

Figure 1. Configural model of Pearce. The network architecture.

senting input pattern $A$. The different gray shadings indicate different degrees of activation.

The configural units are connected to the output unit in the output layer that represents the conditioned response (CR) to the input pattern. At the end of each learning trial, feedback about the presence $\left(\lambda_{\mathrm{US}}\right)$ and absence $\left(\lambda_{\overline{\mathrm{US}}}\right)$ of the unconditioned stimulus (US) is presented.

The processing algorithm in Table 1 comprises three main steps. First, in Step 4, the similarity between the input pattern and the configural patterns of each configural unit is computed. The similarity is defined as the cosine of the angle between the input and the configural vector or, equivalently, as the scalar product between the two normalized vectors. ${ }^{1}$ The activation of a configural unit results by raising the similarity to the power of the specificity parameter $\sigma$. The higher the value of $\sigma$, the greater the rate of decrease of activation with dissimilarity between input and configural pattern. Hence, $\sigma$ influences the size of the active fields of the configural units (i.e., the region of the input space leading to an activation of the unit). It represents cognitive discriminability and memorability of the configural patterns ( $\sigma$ corresponds to the specificity parameter $c$ of ALCOVE; cf. Kruschke, 1992, p. 23). Second, in Step 5, the activation of the output unit is computed as the scalar product of the activation vector of the configural units and the vector of associative weights. Third, in Step 6, the associative weight of the connection from the configural unit with highest activation (i.e., the unit representing the input pattern) to the output unit is modified by means of the Rescorla-Wagner learning rule (Rescorla \& Wagner, 1972).

\section{Application of the Model}

In the following, the results of Experiment 3 of Shanks, Charles, Darby, and Azmi (1998) will be simulated. The CMP in its original formulation by Pearce (1994) is unable to simulate the results (cf. Shanks et al., 1998, p. 1375). However, the CMP, as specified above, with the specificity parameter $\sigma$ regarded as a free parameter, can predict the results.

Description of the experiment. Shanks et al. (1998) investigated configural processing in human discrimination learning. Participants learned associations between different sorts of food and an allergy in a trial-by-trial fashion. A trial consisted of the presentation of either the name of a single food or the names of two foods. Participants had to predict whether an allergy would occur for the presented foods and received feedback. The experimental design is shown in Table 2.

The letters denote foods, whereas the presence and absence of an allergy is indicated by a "+" and a "-" sign, respectively. For example, in Learning Stage 1, there were $15 A C+$ trials (i.e., the foods $A$ and $C$ were present, and the allergy occurred for this combination).

The important comparison concerns participants' responses to the cue combinations $A B$ and $D E$ at the end of learning. In Stage 2, cue $B$ was presented in isolation and reinforced. If patterns are processed in an elementwise fashion, Stage 2 learning should lead to more positive responses for $A B$ than for $D E$ because $B$ is contained in $A B$. Participants' responses at the end of Stage 2 revealed no such increase, indicating that learning of $B$ in Stage 2 does not transfer to the combination $A B$ (cf. Shanks et al., 1998, Figure 3, p. 1362).

Implementation of the model. Two versions of the CMP were implemented on three worksheets within a workbook. In addition, two charts with the models's learning curves (cf. Figures $4 \mathrm{~A}$ and $4 \mathrm{~B}$ ) are contained in the workbook. The first sheet, named "Global Symbols, Input, Results," contains a symbol table, the input patterns and target activations, and the data from which the charts with the learning curves were generated. The second and third sheets, "Simulation 1" and "Simulation 2," contain the computational structures of the two model versions that differ with respect to specificity parameter $\sigma$ only: "Simulation 1," $\sigma=2$; “Simulation 2," $\sigma=10$. 
Table 1

Processing Algorithm of the Configural Model of Pearce (1994)

\begin{tabular}{|c|c|}
\hline Step & Performed Computation \\
\hline 1 & $\begin{array}{l}\text { Initialization: } \\
\text { Label all units of the configural layer as not active, and set all associative weights } \\
\text { to zero. }\end{array}$ \\
\hline 2 & $\begin{array}{l}\text { Presentation of the next input pattern: } \\
\text { Set the activation values of the input units to the values of the normalized input } \\
\text { pattern. }\end{array}$ \\
\hline 3 & $\begin{array}{l}\text { Labeling of the configural unit that corresponds to the input pattern: } \\
\text { Label the configural unit that corresponds to the presented input pattern as being } \\
\text { active, if it has not already been activated. }\end{array}$ \\
\hline \multirow[t]{3}{*}{4} & $\begin{array}{l}\text { Computation of the activation values of the configural units: } \\
\text { Calculate the activation value } a_{j}^{\text {cfg }} \text { of configural unit } j(1 \leq j \leq J) \text { by computing } \\
\text { the scalar product, raised to the power of } \sigma \text { : }\end{array}$ \\
\hline & $\begin{array}{l}\Leftrightarrow \text { unit } j \text { has been labeled active } \\
\Leftrightarrow \text { unit } j \text { has not been labeled active. }\end{array}$ \\
\hline & $\begin{array}{ll}a_{j}^{\text {cfg }} & \text { denotes the activation of configural unit } j: 0 \leq a_{j}^{\text {cfg }} \leq 1 \text {, with the unit } \\
& \text { representing the input pattern being the sole unit with maximal activa- } \\
\text { tion: } a_{j}^{\text {cfg }}=\cos \sigma(\varphi), \text { where } \varphi \text { is the angle between the vectors } \boldsymbol{a}^{\text {in }} \text { and } \\
\\
\boldsymbol{w}_{j}^{\text {cfg. }}\end{array}$ \\
\hline \multirow[t]{2}{*}{5} & $\begin{array}{l}\text { Computation of the activation values of the output unit: } \\
\text { Calculate the activation value } a^{\text {out }} \text { of the output unit according to the formula: }\end{array}$ \\
\hline & $\begin{array}{l}\text { wout denotes the weight of the associative connection from configural unit } \\
j(1 \leq j \leq J) \text { to the output unit. }\end{array}$ \\
\hline \multirow[t]{4}{*}{6} & $\begin{array}{l}\text { Modification of associative weights: } \\
\text { Calculate the weight change } \Delta w_{j}^{\text {out }} \text { of the connection from the configural unit } j \\
\text { representing the input pattern to the output node according to the Rescorla- } \\
\text { Wagner rule: }\end{array}$ \\
\hline & $\Delta w_{j}^{\text {out }}=\alpha_{j} \cdot \beta_{k} \cdot\left(\lambda_{k}-a^{\text {out }}\right)$ \\
\hline & $\begin{array}{l}\text { and add it to the respective associative weight. } \\
\alpha_{j} \quad \text { denotes a saliency parameter specific to the pattern represented by con- } \\
\text { figural unit } j \text { [values used for the present simulations: } \alpha=1,(1 \leq \\
j \leq J) \text { ]. }\end{array}$ \\
\hline & 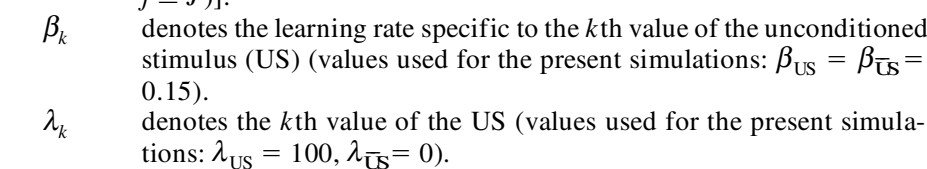 \\
\hline 7 & $\begin{array}{l}\text { Iteration: } \\
\text { If there is another input pattern, go to Step 2, otherwise sтоP. }\end{array}$ \\
\hline
\end{tabular}

Table 3 shows the names that were used within the computational formulas. Names represent cell ranges. To define a name, use the menu command Insert/Name/Define. A menu appears that enables one to enter the name and the associated cell range. The names in Table 3 are divided into two groups: (1) Names of global scope are valid in the whole workbook, and (2) names of local scope are valid within a specific worksheet only. This allows one to use the same name with possibly different values within different sheets. To define a name with local scope, put the name of the worksheet in front of the name, separated by a "!" sign. For example, the name of the specificity parameter $\sigma$ on sheet "Simulation 1 " was specified as 'Simulation 1'!specificity.

For names referring to a single cell, the third column of Table 3, "Value/Cell Range," shows the content of the cell. 
Table 2

Experimental Design of Shanks, Charles, Darby, and Azmi's (1998) Experiment 3

\begin{tabular}{ccccc}
\hline \multicolumn{2}{c}{ Learning Stage 1 } & & \multicolumn{2}{c}{ Learning Stage 2 } \\
\cline { 5 - 5 } Pattern & $\begin{array}{r}\text { Number } \\
\text { of Trials }\end{array}$ & & Pattern & $\begin{array}{c}\text { Number } \\
\text { of Trials }\end{array}$ \\
\hline$A+$ & 15 & & $B+$ & 10 \\
$A B-$ & 15 & & $G H+$ & 10 \\
$A C+$ & 15 & & $I J-$ & 10 \\
$D+$ & 15 & & \\
$D E-$ & 15 & & \\
$D F+$ & 15 & & \\
\hline
\end{tabular}

For example, "minactivity" refers to the cell that contains the value 0.99 . In all computational expressions containing the name "minactivity," this value enters the computation. If a name refers to a cell range with multiple cells, the third column of Table 3 shows the address of the upper left and the lower right corner of the range. For example, "CS" refers to the table with the normalized input patterns that is located in cell range C49:H55 (letters denote columns, and numbers denote rows). Names with local scope are defined twice: on sheet "Simulation 1," and on sheet "Simulation 2." However, except for the specificity parameter $\sigma$, the values and ranges (the names refer to) are identical.

Figure 2 shows an extract of the worksheet "Global Symbols, Input, Results." The row indices, 36-60, and the column indices, A-H, enable the localization of the different structures. Three tables are shown: (1) The table with the non-normalized input patterns as rows (cell range C40:H46), (2) the table "CS" with the normalized input patterns as rows (cell range C49:H55), and (3) the table
"US" with target activations representing positive and negative feedback (cell range D59:D60). There are no input patterns corresponding to the cue combinations $G H$ and $I J$ that were used by Shanks et al. (1998) as filler stimuli in Stage 2 (cf. Table 2). These stimuli were dropped because they do not influence the model's response to $A B$ and $D E$ at the end of learning.

The formula in the box beneath the sheet is an array formula, as indicated by the curly braces. To specify an array formula, perform the following steps: (1) Select the target range (in the present case, C55:H55), (2) enter the formula, and (3) terminate the input with the key combination $\mathrm{Ctrl}+$ Shift + Enter (Macintosh, $\mathrm{Cmd}+$ Enter). The program adds the curly braces (Excel 5 [for Windows] and Excel 98 [for Macintosh] do not allow the user to enter array formulas from within the formula wizard). The shown array formula normalizes the input pattern $D F$ by executing the following computations: (1) The subexpression " $\mathrm{C} 46: \mathrm{H}_{4} 6^{\wedge} 2$ " computes a row vector with the squares of the input row vector $\mathrm{C} 46: \mathrm{H} 46$ as components (" $\wedge$ " is the power operator). (2) The worksheet function SUM( ) computes the sum of the components of this vector. (3) The function SQRT( ) calculates the square root of this sum. The result corresponds to the (Euclidean) length of the input vector C46:H46. (4) Each component of the input vector is divided by its length (cf. note 1). The arrows pointing to the cells C55:H55 with the normalized vector indicate that each cell contains the same expression. These cells constitute a row vector, and none of them can be modified in isolation.

Each row of table "CS" contains the same array formula, yet with a different reference. For example, row 49 contains the expression " $\{=\mathrm{C} 40: \mathrm{H} 40 / \mathrm{SQRT}(\mathrm{SUM}(\mathrm{C} 40$ :

Table 3

Configural Model of Pearce: Symbols and Corresponding Names Used Within the Workbook for Implementing the Models

\begin{tabular}{|c|c|c|c|}
\hline Symbol & Name & $\begin{array}{c}\text { Value / } \\
\text { Cell Range }\end{array}$ & Description \\
\hline \multicolumn{4}{|c|}{ Names with Global Scope } \\
\hline & minactivity & 0.99 & $\begin{array}{l}\text { If the activity of a configural unit is greater than } \\
\text { this value, the unit is maximally activated }\end{array}$ \\
\hline & $\mathrm{CS}$ & C49:H55 & Table with normalized input patterns \\
\hline & US & D59:D60 & Table with values of US \\
\hline & Sim1 & AH20:AH139 & $\begin{array}{l}\text { Column containing the activity values of the out- } \\
\text { put unit (Simulation } 1 \text { ) }\end{array}$ \\
\hline & $\operatorname{Sim} 2$ & AH20:AH139 & $\begin{array}{l}\text { Column containing the activity values of the out- } \\
\text { put unit (Simulation } 2 \text { ) }\end{array}$ \\
\hline \multicolumn{4}{|c|}{ Names with Local Scope } \\
\hline$\alpha$ & alpha & 1.00 & Saliency parameter \\
\hline$\beta$ & beta & 0.15 & Learning rate \\
\hline \multirow[t]{2}{*}{$\sigma$} & specificity & $\begin{array}{l}2.00 \\
10.00\end{array}$ & $\begin{array}{l}\text { Specificity parameter: The first entry }(2.0) \text { was } \\
\text { used in Simulation } 1 \text {, and the second entry }(10.0) \\
\text { was used in Simulation } 2\end{array}$ \\
\hline & $\mathrm{S}$ & B20:B139 & Index into the stimulus table CS \\
\hline$\lambda$ & lambda & D20:D139 & Index into the response table US \\
\hline$a^{\text {out }}$ & $\begin{array}{l}\text { cfg } \\
\text { aout }\end{array}$ & $\begin{array}{l}\text { F18:L18 } \\
\text { AH20:AH139 }\end{array}$ & $\begin{array}{l}\text { Index for addressing patterns of configural units } \\
\text { Column containing the activity values of the out- } \\
\text { put unit }\end{array}$ \\
\hline
\end{tabular}




\begin{tabular}{|c|c|c|c|c|c|c|c|c|}
\hline & A & B & C & D & E & $F$ & G & H \\
\hline 36 & \multicolumn{5}{|c|}{ THE TABLE WITH INPUT VECTORS } & & & \\
\hline 37 & & & & & & & & \\
\hline 38 & \multirow{2}{*}{\multicolumn{2}{|c|}{ Pattern }} & \multicolumn{6}{|c|}{ Not normalized } \\
\hline 39 & & & $\mathbf{A}$ & B & C & D & $\mathbf{E}$ & $\mathbf{F}$ \\
\hline 40 & 1 & $A$ & 1. & 0 & 0 & 0 & 0 & 0 \\
\hline 41 & 2 & B & 0 & 1 & 0 & 0 & 0 & 0 \\
\hline 42 & 3 & $A B$ & 1 & 1 & 0 & 0 & 0 & 0 \\
\hline 43 & 4 & $A C$ & 1 & 0 & 1 & 0 & 0 & 0 \\
\hline 44 & 5 & D & 0 & 0 & 0 & 1 & 0 & 0 \\
\hline 45 & 6 & DE & 0 & 0 & 0 & 1 & 1 & 0 \\
\hline 46 & 7 & DF & 0 & 0 & 0 & 1 & 0 & 1 \\
\hline 47 & \multirow{2}{*}{\multicolumn{2}{|c|}{ Pattern }} & \multicolumn{6}{|c|}{ Normalized } \\
\hline 48 & & & A & B & C & D & $\mathbf{E}$ & $\mathbf{F}$ \\
\hline 49 & 1 & $A$ & 1.000 & 0.000 & 0.000 & 0.000 & 0.000 & 0.000 \\
\hline 50 & 2 & $\mathrm{~B}$ & 0.000 & 1.000 & 0.000 & 0.000 & 0.000 & 0.000 \\
\hline 51 & 3 & $A B$ & 0.707 & 0.707 & 0.000 & 0.000 & 0.000 & 0.000 \\
\hline 52 & 4 & $A C$ & 0.707 & 0.000 & 0.707 & 0.000 & 0.000 & 0.000 \\
\hline 53 & 5 & D & 0.000 & 0.000 & 0.000 & 1.000 & 0.000 & 0.000 \\
\hline 54 & 6 & $D E$ & 0.000 & 0.000 & 0.000 & 0.707 & 0.707 & 0.000 \\
\hline 55 & 7 & $\mathrm{DF}$ & 0.000 & 0.000 & 0.000 & 0.707 & 0.000 & 0.707 \\
\hline 56 & & & $t$ & 4 & 4 & 4 & 4 & 4 \\
\hline 57 & \multicolumn{4}{|c|}{ THE TABLE WITH VALUES OF US } & & & & \\
\hline 58 & & & & & & & & \\
\hline 59 & & 1 & $\lambda_{1}$ & 100 & & & & \\
\hline 60 & & 2 & $\lambda_{2}$ & 0 & & & & \\
\hline
\end{tabular}

Figure 2. Configural model of Pearce. Extract of the worksheet "Global Symbols, Input, Results."

$\left.\left.\left.\mathrm{H}_{40}{ }^{\wedge} 2\right)\right)\right\}$ " for normalizing input pattern $A$. The formula for the first entry must be specified only. Then, it can be copied to the other rows, resulting in an automatic adjustment of the row references within the formulas. To copy the formula of the first row, select the source range C49:H49 and pull the selected range over the other rows by means of the mouse handle (i.e., the small black square at lower right corner of the selected range).

Figures $3 \mathrm{~A}, 3 \mathrm{~B}$, and $3 \mathrm{C}$ show extracts of the worksheet "Simulation 1" with the model's computational structures. On the sheet, these structures are arranged side by side with each row containing the computations of one trial, beginning with row 20 that contains the computations of the first trial.

In Figure 3A, column A contains a trial counter that is not used for the computation. Column B contains index numbers for retrieving the input patterns within the different trials from the table "CS." Column C contains a description of the trial type that is also of documentary purpose only. The index numbers in column D are used for retrieving target activations from the table "US." Column E contains a learning flag. The associative weights within a trial are modified only if the value of the learning flag in the previous trial is greater than zero. Columns F-L con- tain the activations of the seven configural units without taking into account whether a unit had already been assigned the status "active." The numbers in the cell range F18:L18 are used for retrieving the configural patterns from the table "CS."

The formula in the box below the sheet computes the activations of the configural units in Trial 6. Again, the arrows indicate that the cells F25-L25 contain the same expression. The formula is not an array formula (no curly braces). The function SUMPRODUCT( ) takes as input two vectors of the same dimension and computes the scalar product. In the shown expression, the two vectors are given by the subexpressions "INDEX $(\mathrm{CS}, \mathrm{S}, 0)$," representing the input vector of Trial 6, and "INDEX(CS, cfg, 0)" that represents the configural vectors of the configural units. Both INDEX( ) functions access the normalized vectors in the table "CS." In the present case, they take the following three input arguments. (1) The first argument is the cell range to be accessed: the table "CS" (cell range C49:H55 on the sheet "Global Symbols, Input, Results"). (2) The second argument is the number of the row to be addressed within the given range: "S" (cell range B20: B139) for the first INDEX ( ) function, and "cfg" (cell range F18:L18) for the second INDEX( ) function. The cell range 


\begin{tabular}{|c|c|c|c|c|c|c|c|c|c|c|c|c|}
\hline 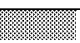 & A & $\mathrm{B}$ & (). & D & E & 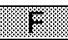 & C. & II) & 1 & J & L. & 1 \\
\hline I\% & & & & & & \multicolumn{7}{|c|}{ Similarity specificity } \\
\hline 18 & & & & & & 1 & 2 & 3 & 4 & 5 & 6 & 7 \\
\hline 19 & Trial & Stimulus & Type & $\lambda$ & Iflag & A & $\mathrm{B}$ & $A B$ & $A C$ & D & $D E$ & DF \\
\hline 20 & 1 & 1 & $A+$ & 1 & 1 & 1.00 & 0.00 & 0.50 & 0.50 & 0.00 & 0.00 & 0.00 \\
\hline $21: 1$ & 2 & 3 & $A B-$ & 2 & 1 & 0.50 & 0.50 & 1.00 & 0.25 & 0.00 & 0.00 & 0.00 \\
\hline 22 & 3 & 4 & $A C+$ & 1 & 1 & 0.50 & 0.00 & 0.25 & 1.00 & 0.00 & 0.00 & 0.00 \\
\hline 23 & 4 & 5 & $D+$ & 1 & 1 & 0.00 & 0.00 & 0.00 & 0.00 & 1.00 & 0.50 & 0.50 \\
\hline 24. & 5 & 6 & DE- & 2 & 1 & 0.00 & 0.00 & 0.00 & 0.00 & 0.50 & 1.00 & 0.25 \\
\hline 25. & 6 & 7 & $\mathrm{DF}+$ & 1 & 1 & 0.00 & 0.00 & 0.00 & 0.00 & 0.50 & 0.25 & 1.00 \\
\hline & & & & & & & & & 10 & & & icity \\
\hline
\end{tabular}

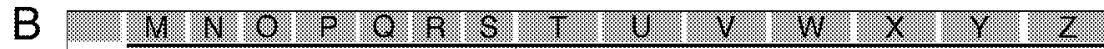

\begin{tabular}{|c|c|c|c|c|c|c|c|c|c|c|c|c|c|c|}
\hline 17 & \multicolumn{10}{c|}{ Activity Flags } & \multicolumn{1}{c|}{ Activity of Configural Units } \\
\hline 18 & 1 & 2 & 3 & 4 & 5 & 6 & 7 & 1 & 2 & 3 & 4 & 5 & 6 & 7 \\
\hline 19 & $\mathrm{~A}$ & $\mathrm{~B}$ & $\mathrm{AB}$ & $\mathrm{AC}$ & $\mathrm{D}$ & $\mathrm{DE}$ & $\mathrm{DF}$ & $\mathrm{A}$ & $\mathrm{B}$ & $\mathrm{AB}$ & $\mathrm{AC}$ & $\mathrm{D}$ & $\mathrm{DE}$ & $\mathrm{DF}$ \\
\hline 20 & 1 & 0 & 0 & 0 & 0 & 0 & 0 & 1.00 & 0.00 & 0.00 & 0.00 & 0.00 & 0.00 & 0.00 \\
\hline 21 & 1 & 0 & 1 & 0 & 0 & 0 & 0 & 0.50 & 0.00 & 1.00 & 0.00 & 0.00 & 0.00 & 0.00 \\
\hline 22 & 1 & 0 & 1 & 1 & 0 & 0 & 0 & 0.50 & 0.00 & 0.25 & 1.00 & 0.00 & 0.00 & 0.00 \\
\hline 23 & 1 & 0 & 1 & 1 & 1 & 0 & 0 & 0.00 & 0.00 & 0.00 & 0.00 & 1.00 & 0.00 & 0.00 \\
\hline 24 & 1 & 0 & 1 & 1 & 1 & 1 & 0 & 0.00 & 0.00 & 0.00 & 0.00 & 0.50 & 1.00 & 0.00 \\
\hline 25 & 1 & 0 & 1 & 1 & 1 & 1 & 1 & 0.00 & 0.00 & 0.00 & 0.00 & 0.50 & 0.25 & 1.00 \\
\hline
\end{tabular}

$\{=I F((F 25: L 25>=$ minactivity $)+(M 24: S 24>=$ minactivity $)>0,1,0)\} \quad\{=F 25: L 25 * M 25: S 25\}$

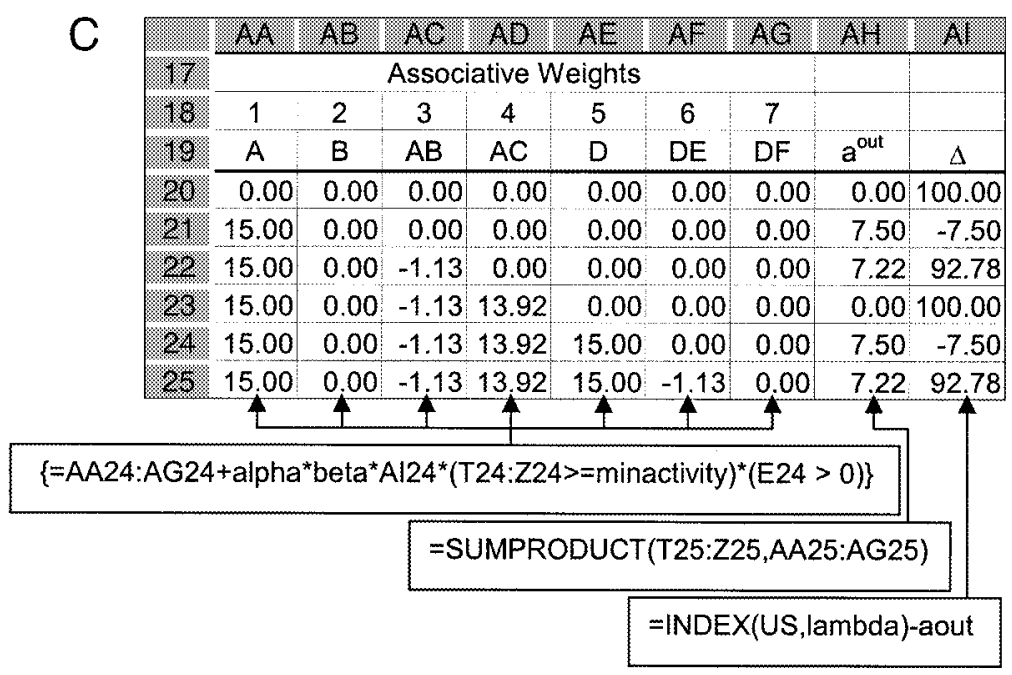

Figure 3. (A) Configural model of Pearce. Extract of the worksheet "Simulation 1," Part 1: Computation of similarity between configural units and the input pattern set to the power of the decay parameter. (B) Configural model of Pearce. Extract of the worksheet "Simulation 1," Part 2: Computation of activity flags and activation of the configural units. (C) Configural model of Pearce. Extract of the worksheet "Simulation 1," Part 3: Computation of activation of the output unit and of the associative weights.

that "S" refers to contains the stimulus numbers of the 120 learning trials. The value of " $\mathrm{S}$ " in row 25 (i.e., the row with the shown expression) is 7 (cell B25 in Figure 3A). Thus, the seventh row of table "CS," containing the normalized input pattern $D F$, is addressed (cf. Figure 2). The cell range that "cfg" refers to holds the index numbers of the configural units. The value of "cfg" in column $F$ is 1 . Thus, the second INDEX( ) function in this column addresses the first row of table "CS" with the normalized input pattern $A$. Column $\mathrm{F}$ contains, therefore, the activa- 
tions of the configural unit representing pattern $A$. Analogously, columns H-L contain the activations of the configural units representing the patterns $B-D F$. (The following general principle underlies the usage of the names denoting row and column vectors, such as "cfg" and "S," within a formula that is not an array formula: In case of column vectors, the value in the same row as the formula is used. Formulas in the same row as the first component use the first entry of the vector, formulas in the same row as the second component use the second entry, and so on. Formulas outside the row range of the name lead to the error message "\#VALUE" indicating an erroneous value. The same principle applies to row vectors. In this case, the name is replaced by the value in the respective column.) (3) The third argument of the INDEX( ) function is the column index: The number 0 (both INDEX( ) functions) indicates that the whole row is retrieved, and not a single cell only.

In Figure 3B, columns $\mathrm{M}-\mathrm{S}$ contain the activity flags of the seven configural units. The flags indicate the activity status of the units $(1=$ active $)$. Once activated, a unit stays active. After the first learning block, all configural units are active except for the one representing stimulus $B$ that was not yet presented. Columns T-Z contain the activations of the configural units computed according to equation T1 of Table 1 .

The array function in the box on the left computes the values of the activity flags in Trial 6 . The IF( ) function takes three arguments: (1) a conditional expression, (2) the return value of the function if the conditional expression evaluates to TRUE (T), and (3) the return value if the conditional expression evaluates to FALSE $(\mathrm{F})$. In the present case, the formula is an array formula working on row vectors. As a result, all involved quantities are row vectors. The conditional expression of the IF( ) function, "(F25:L25>= minactivity $)+(\mathrm{M} 24: \mathrm{S} 24>=$ minactivity $)>0$ " represents the Boolean vector [T F T T T T T]. It results from the following computations. (1) The subexpression "F25:L25>= minactivity" tests for each component of the row vector in cells F25:L25 whether it is greater than "minactivity"that is, whether it represents input pattern $D F$ (due to rounding errors, the expression "F25:L25>=1.0" does not work properly). The resulting Boolean vector [F F F F F F T] indicates that the last configural unit $D F$ represents the actual input. (2) "M24:S24>= minactivity" determines which configural units had already been active in the previous trial. The resulting Boolean vector is [T F T T T T T]. (3) The two Boolean vectors are added, leading to an automatic conversion: TRUE to 1 , FALSE to 0 . The resulting vector is [ $\left[\begin{array}{lllllll}1 & 0 & 1 & 1 & 1 & 1 & 2\end{array}\right]$. (4) Each component of this vector is tested whether it is greater than zero. The resulting Boolean vector [ $\mathrm{T} F \mathrm{~F} \mathrm{~T} \mathrm{~T} \mathrm{~T} \mathrm{~T}$ ] is converted to $\left[\begin{array}{lllllll}1 & 0 & 1 & 1 & 1 & 1 & 1\end{array}\right]$ because the second argument of the $\operatorname{IF}()$ function, 1 , replaces TRUE, and the third argument, 0 , replaces FALSE. Thus, in Trial 6, all configural units, except for the one representing stimulus $B$, are active.

The array formula uses an expansion mechanism that is related to the usage of array formulas. The conditional expressions "F25:L25>= minactivity" and "M24:S24>= minactivity" perform comparisons between vectors and the scalar "minactivity." To this end, the program expands the scalar to the vector [0.99 0.990 .990 .990 .990 .990 .99 ], compares the components of the two vectors, and returns a Boolean vector representing the results of the single comparisons. Similarly, the second and third arguments of the IF() function are expanded to the vectors

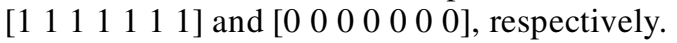

The array formula in the box assigned to the range T25:Z25 computes the activation vector of the configural layer (in Trial 6) by componentwise multiplication of the vector of computed similarities, raised to the power of the specificity parameter, and the vector with the values of the activity flags.

In Figure 3C, columns AA-AG contain the associative weights of the connections from the configural units to the output unit, column AH contains the activations of the output unit, and column AI contains the delta values (i.e., the difference between the output and target activation).

The array formula in the box assigned to the cell range AA25:AG25 computes the row vector of associative weights in Trial 6. It contains two summands: (1) "AA24: AG24" represents the weight vector of the previous trial, and (2) "alpha*beta*AI24*(T24:Z24>= minactivity)* (E24 > 0)" represents the vector [ [ $\left.\begin{array}{lllllll}0 & 0 & 0 & 0 & 0 & -1.13 & 0\end{array}\right]$ of weight changes. The subexpressions: "alpha*beta*AI24* (T24:Z24>= minactivity)" implements the RescorlaWagner rule (cf. Table 1, Equation T3), using the delta value of Trial 5 that is located in cell AI24. The subexpression "(T24:Z24>=minactivity)," computing the vector [F F F F F F F], guarantees that the weight of the configural unit representing the input pattern $D E$ in Trial 5 is modified only. The subexpression "(E24>0)" for testing whether the learning flag had been set in Trial 5 represents the vector [T T T T T T T].

The expression in the middle box computes the activation of the output unit as the scalar product of the activation vector T25:Z25 of the configural layer and the vector AA25:AG25 of associative weights (cf. Table 1, Equation T2). The expression in the right box computes the delta value. The INDEX( ) function, retrieving the target activation from the table "US," contains two input arguments only, because "US" refers to a column vector.

\section{Results of the Simulation}

Figures 4A and 4B show the development of the output activations for the different input stimuli in the two learning stages. Figure 4A depicts the simulation results for $\sigma=2$; Figure 4B depicts those for $\sigma=10$. The other parameters were identical in both simulations: $\alpha=1$, $\beta=0.15$. In Stage 1,15 blocks, with 6 stimuli per block, were presented (cf. Table 2, left column), always in the same order as in Table 2. Stage 2 consisted of $10 B+$ trials.

With $\sigma=2$, the $B+$ trials in Stage 2 result in a higher activation for $A B$ than for $D E$ at the end of learning, which is in opposition to the experimental finding of no difference. With $\sigma=10$, the activations for $A B$ and $D E$ at the 

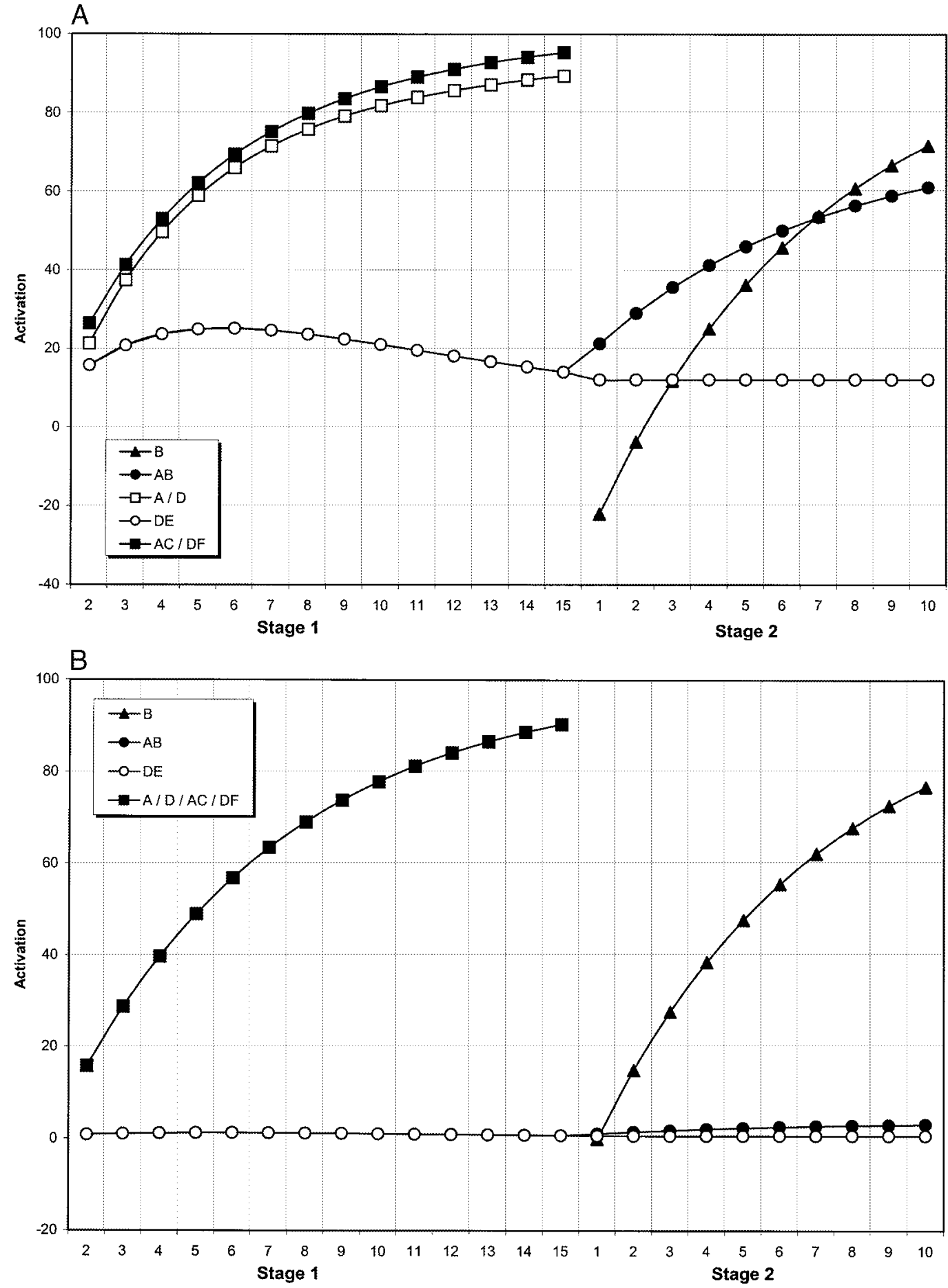

Figure 4. (A) Configural model of Pearce. Activation of the output unit for different input patterns as a function of learning with specificity parameter $\sigma=2$. (B) Configural model of Pearce. Activation of the output unit for different input patterns as function of learning with specificity parameter $\sigma=10$.

end of learning are practically identical. This demonstrates that the CMP with a high value of the specificity parameter $\sigma$ can reproduce the experimental results of Shanks et al. (1998).

\section{Discussion}

The following aspects related to the present implementation will be discussed: First is a summary of the organization of the model within the workbook. Second is a 
consideration of different methods for implementing iterated models. Third is a discussion of names and cell references. Fourth is a treatment of array formulas and the automatic expansion mechanism.

Organization of the model within the workbook. The model was distributed on two spreadsheets: (1) a sheet containing the input and target patterns (cf. Figure 2), and (2) a sheet with the computational structures (cf. Figure $3 \mathrm{~A}, 3 \mathrm{~B}$, and $3 \mathrm{C}$ ). The latter consist of formulas linked together in a left-to-right fashion: Names and references within formulas generally refer to cells on the left of the same row. This organization reflects the propagation of activation through the network within a single trial. The modification of weights follows a different pattern: References refer to cells in the previous row (cf. Figure 3C), reflecting the fact that the weights can be modified only after having accomplished the propagation of activation.

With neural networks comprising many layers, it might be useful to use one sheet per layer. The linkage of computational structure works in the same way, independent of whether a referenced cell range is on the same sheet or on a different one. (Usually, cell ranges are entered into a formula by selecting them with the mouse. If the selected range is located on a different sheet, the program puts the worksheet name in front of the cell reference, thus ensuring that the cells on the correct sheet are addressed. If a name is defined for the selected range, the program inserts the name instead of the reference into the formula.)

Linked computational structures can give rise to $\mathrm{circu}$ lar references - that is, formulas referring to their own outcome, either directly or indirectly via other formulas. For example, inserting " $=\mathrm{B} 1+1 "$ into cell A1 and " $=\mathrm{A} 1+1$ " into cell $\mathrm{B} 1$ results in a circular reference: Evaluating the formula in cell A1 requires knowledge of the result in cell B1, which in turn is based on the result in cell A1. With automatic recomputation (the default mode of computation), a circular reference generates an error message, and the program displays arrows pointing to the critical cells. In addition, it provides tools for identifying the cells making up the circular reference. If, in the present implementation of the CMP, the formulas for weight modification erroneously referred to cells within the same row instead of the previous one (cf. Figure 3C), the following circular reference would occur: Associative weights $($ trial $t) \rightarrow$ output activation (trial $t) \rightarrow$ delta (trial $t) \rightarrow$ associative weights (trial $t$ ) (the arrows indicate "enters the computation of"). Computing the weight modification in trial $t$ on the basis of the delta value of trial $t-1$ precludes this circular reference.

Iterated models and iterated worksheets. Like most neural networks, the CMP is an iterated model: The processing consists of a number of iterations composed of the same basic steps (cf. Table 1). This iterative structure gave rise to the following method of implementation: The computational formulas of the first iteration/trial were specified only. Then, they were copied to the other rows. This works because copying a formula results in an adjustment of the cell references. For example, copying for- mula " $=\mathrm{A} 1+1$ " in cell $\mathrm{B} 1$ to cell $\mathrm{C} 3$ results in " $=\mathrm{B} 3+1 "$ in cell $\mathrm{C} 3$ : The row index is incremented by two because cell C3 is two rows below B 1 , whereas the column index is incremented by one because $\mathrm{C} 3$ is one column to the right of B1. Fixed references, indicated by a "\$" sign in front of the reference, suppress the automatic adjustment. For example, copying " $=\$ A 1+1 "$ (column reference fixed) from cell B1 to cell C 3 results in " $=\$ A 3+1$." Copying "= $\mathrm{A} \$ 1+1$ " (row reference fixed) from $\mathrm{B} 1$ to $\mathrm{C} 3$ results in " $=\mathrm{B} \$ 1+1$." Finally, copying " $=\$ \mathrm{~A} \$ 1+1$ " does not change the formula.

Iterated worksheets provide a more efficient method for implementing iterated models. This approach requires two basic steps: (1) Set the calculation mode from automatic to manual mode (menu command Tools/Options/ Calculation), and (2) use circular references for implementing an iteration (for details, see Orvis, 1996; the Web site with the models also contains an iterated worksheet version of the CMP).

Using names and references. The presented implementation predominately uses names instead of cell references. By default, names are equivalent to fixed references (it is possible, but not recommended, to define names as variable references). Thus, replacing the names with the fixed references (e.g., "cfg" with "\$F\$18:\$L\$18”) does not change the computations. Names enhance the comprehensibility of an implementation. They can also be defined after the specification of the formulas. A program's utility enables the automatic replacement of cell references within formulas with the assigned names (menu command Insert/Name/Apply).

Array formulas and automatic expansion. Array formulas enable the processing of vectors and matrices in two different ways: (1) Mathematical functions within array formulas are applied to each entry of a matrix/vector, and (2) worksheet functions for performing matrix operations, such as MMULT $(X, Y)$ for multiplying matrices " $\mathrm{X}$ " and "Y," work properly only within array formulas.

The utility of array formulas is enhanced by the automatic expansion mechanism that was used above for computing activity flags (cf. Figure 3B) and weight modifications (cf. Figure 3C). In both cases, scalars were expanded to row vectors with identical components. The expansion mechanism works more generally. For example, let " $z$ " be a row vector of dimension $m$, "s" a column vector of dimension $n$, "k" a scalar, and "M" an $n \times m$ matrix. The processing of the array formula " $\{\mathrm{M}+\mathrm{z}+\mathrm{s}+\mathrm{k}\}$ " results in the following expansions: (1) " $\mathrm{z}$ " is expanded to an $n \times m$ matrix with each row identical to " $\mathrm{z}$," (2) " $\mathrm{s}$ " is expanded to an $n \times m$ matrix with each column identical to "s," and (3) " $\mathrm{k}$ " is expanded to an $n \times m$ matrix with each entry identical to " $k$." The expansion mechanism fails if the number of entries of the column vector "s" differs from the number of rows of "M" or if the number of entries of the row vector " $z$ " is different from the number of columns of "M".

The presented method for implementing the CMP by means of spreadsheets applies to other models as well, 
such as the Rescorla-Wagner model or ALCOVE (Macho, in press; workbooks with these models can be retrieved from http://www.unifr.ch/psycho/general/macho/neural. $\mathrm{htm})$. The next example illustrates two further spreadsheet facilities: the integrated optimizer and the library of statistical worksheet functions. They are helpful for implementing statistical models.

\section{MULTINOMIAL PROCESSING TREE SIGNAL DETECTION MODEL}

The multinomial processing tree signal detection model (MPTSD) is a hybrid model that integrates a multinomial processing tree component and a signal detection component.

Multinomial processing trees simulate distributions of response categories by assuming hypothetical (cognitive) processes that are arranged in a tree structure with the observed categories as leaves. The model parameters represent the probabilities of the various processes to become active (see, e.g., Hu \& Batchelder, 1994; Riefer \& Batchelder, 1988). Multinomial processing trees were applied in various areas, such as learning (Riefer \& Batchelder, 1988), source monitoring (Batchelder \& Riefer, 1990; Bayen, Murnane, \& Erdfelder, 1996; Bayen, Nakamura, Dupuis, \& Yang, 2000; Klauer \& Wegener, 1998), hindsight bias (Erdfelder \& Buchner, 1998a), and recognition memory (Buchner, Erdfelder, \& Vaterrodt-Plünnecke, 1995).

Signal detection models incorporate three basic assumptions (see, e.g., Macmillan \& Creelman, 1991): (1) The stimulus set is partitioned into exhaustive and nonoverlapping classes. (2) A presented stimulus is mapped into a decision space made up of different regions. Each region is assigned to a response that is elicited if a stimulus is mapped into the corresponding region. (3) Probability distributions, one for each stimulus class, specify the probabilities of mapping the stimuli of the respective class into different areas of the decision space. Figure 5 illustrates these features for the signal detection component of the MPTSD.

The model simulates distributions of confidence judgments in memory recognition tasks. Thus, the stimulus set consists of two classes: old and new items. The decision space is a one-dimensional familiarity space that is partitioned into six regions by the decision criteria $c_{1}, c_{2}, \ldots$, $c_{5}$. If a presented memory probe is mapped into the region on the left of $c_{1}$, Response $\mathrm{R}=1$ (= sure new) is elicited, an item falling between $c_{1}$ and $c_{2}$ results in $\mathrm{R}=2$, and so on. The two normal density curves specify the probabilistic mapping for new and old items. For example, the probability of mapping a new stimulus to the region that is assigned to $\mathrm{R}=3$ corresponds to the area under the left density curve between the vertical lines $c_{2}$ and $c_{3}$.

In the following, I first describe the logic of the experiments of Yonelinas (1994). This is followed by the specification of the model.

\section{Description of the Experiments}

Yonelinas's (1994) experiments used Jacoby's (1991) process dissociation method: In the study phase, participants received two lists of words, L1 and L2, that were studied one after another. In the testing phase, two test lists were presented, containing the items from L1 and L2 as well as new distractor items. In the first test, participants had to identify L1 items as "old" and L2 items as well as the new distractor items as "new." For the second test list, L2 items had to be identified as "old," and L1 items as well as distractor items had to be identified as "new." The test items were, therefore, made up of three types: (1) Inclusion items are study items that had to be identified as "old" (L1 items in the first test and L2 items in the second test); (2) exclusion items are study items that had to be identified as "new" (L2 items in the first test and L1 items in the second test); (3) distractoritems are items not studied that had to be identified as "new." Participants responded on a 6-point scale ranging from sure new $(\mathrm{R}=1)$ to sure old $(\mathrm{R}=6)$.

\section{Description of the Model}

Yonelinas (1994) assumes that item recognition involves two processes: (1) An all-or-none process of conscious recollection (an item is either recollected or not), and (2) a process of familiarity assessment that can be modeled as a Gaussian signal detection process with equal variance of the distributions of new and old items (cf. Figure 5). This two-process model should account for the finding of Ratcliff, Sheu, and Gronlund (1992) that the variance of the distribution of old items is about 1.25 the variance of the distribution of new items. Despite a close correspondence between data and model predictions (Yonelinas, 1994; Yonelinas \& Jacoby, 1996), the model does not fit the data of Yonelinas's (1994) Experiments 1 and 2 (Erdfelder \& Buchner, 1998b).

The MPTSD in Figure 6 extends Yonelinas's model by relaxing the restriction that, in case of conscious recollection, $\mathrm{R}=6$ (sure old) for inclusion and $\mathrm{R}=1$ (sure new) for exclusion items is chosen only.

The model assumes that inclusion and exclusion items are consciously recollected with probability $r$. Recollecting an inclusion item results in Response $\mathrm{R}=6, \mathrm{R}=5$, and $\mathrm{R}=4$, respectively, with probabilities $j_{3}, j_{2}$, and $j_{1}$. Recollection of exclusion items results in $\mathrm{R}=1, \mathrm{R}=2$, and $\mathrm{R}=3$, respectively, with probabilities $j_{3}, j_{2}$, and $j_{1}$. Distractor items cannot be consciously recollected.

Judgments for nonrecollected items are based on familiarity assessment that is modeled as a signal detection process: The probabilistic mapping of exclusion and inclusion items into the familiarity space is specified by the distribution for old items. The mapping of distractor items is given by the density function for new items. Table 4 shows the model equations specifying, for each of the three item types, the distribution of response categories.

For example, the equation specifying the probability $P(\mathrm{R}=4)$ for inclusion items contains two summands cor- 


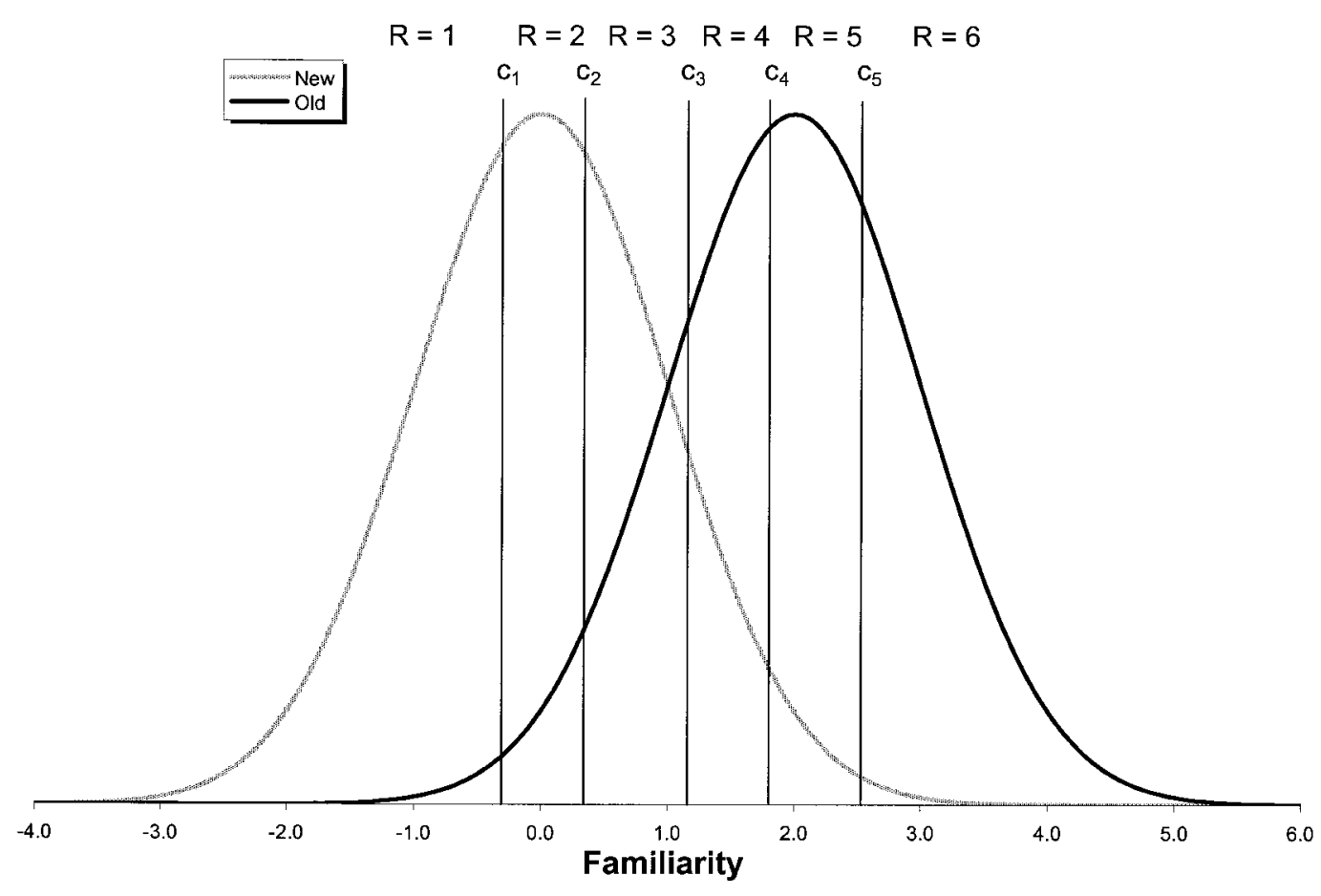

Figure 5. Gaussian signal detection model with equal variance for predicting recognition judgments.

responding to the two branches (= independent ways) leading to $\mathrm{R}=4$ : (1) $r \cdot j_{1}$, is the joint probability that the item is recollected and $\mathrm{R}=4$ is chosen; $(2)(1-r) \cdot\left[\Phi\left(c_{4}-\right.\right.$ $\left.\left.d^{\prime}\right)-\Phi\left(c_{3}-d^{\prime}\right)\right]$ is the joint probability that the item is not recollected and that it is mapped into the region between the decision criteria $c_{3}$ and $c_{4} . \Phi()$ denotes the standard cumulative normal distributionfunction, and $d^{\prime}$ is the mean of the distribution of old items. The distribution of the new items is centered at zero.

The MPTSD contains 9 free parameters for explaining 15 independent data points. The processing tree component comprises three parameters: the probability $r$ of conscious recollection, and the conditional probabilities $j_{1}$ and $j_{2}$ of choosing different response categories in case of conscious recollection (due to the restriction $j_{1}+j_{2}+j_{3}=1$, there are two independent parameters only). The signal detection component contains the decision criteria $c_{1}, c_{2}, \ldots, c_{5}$, and the distance $d^{\prime}$ between the means of the two normal distributions. An extended version, the MPTSD(Var) that assumes unequal variance of the familiarity distributions for old and new items, includes an additional parameter: the standard deviation $\sigma$ of the distribution of new items. The more restricted model of Yonelinas (1994) is a special case of the MPTSD that results by fixing parameters: $j_{3}=$ $1, j_{1}=j_{2}=0$.

\section{Implementation of the Models}

Table 5 presents the symbols and the worksheet names with the corresponding cell ranges. All names are of local scope.
Figures 7A, 7B, and 7C present extracts of the worksheet for simulating the data for inclusion, exclusion, and distractor items, respectively, of Experiment 3 of Yonelinas (1994).

Column A contains the numbers of the six response categories. Columns B and C contain the observed frequencies of the six categories for weak and strong items, respectively (the study time was $1 \mathrm{sec}$ for weak items and $3 \mathrm{sec}$ for strong items). Columns $\mathrm{E}$ and $\mathrm{F}$ contain the predicted frequencies of the MPTSD for weak and strong items, respectively. Column G contains the predicted counts of the MPTSD(Var) for modeling the strong items. Columns I-J contain the corresponding predicted probabilities.

The array formulas in the boxes on the left are located in columns $\mathrm{E}$ (first box) through $\mathrm{G}$ (third box). They compute column vectors of predicted frequencies by multiplying the column vector of predicted probabilities with the sum of the observed frequencies. For example, the formula in the first box in Figure 7A performs a multiplication of the sum of observed frequencies for weak items (cell range B97:B102) and the vector of predicted probabilities (cell range H97:H102). The resulting column vector of predicted frequencies is located in the cell range E97:E102.

The formulas in the boxes assigned to column $\mathrm{H}$ of Figures $7 \mathrm{~A}, 7 \mathrm{~B}$, and $7 \mathrm{C}$ implement the 18 model equations of Table 4 for predicting the response distributions of the weak items. The parameters $r, d^{\prime}, c_{1}, c_{2}, \ldots, c_{5}, j_{1}, j_{2}$, and $j_{3}$ were named "rweak," "dweak," "cweak1," "cweak2," ..., “cweak5," "jweak1," "jweak2," and “jweak3." The 

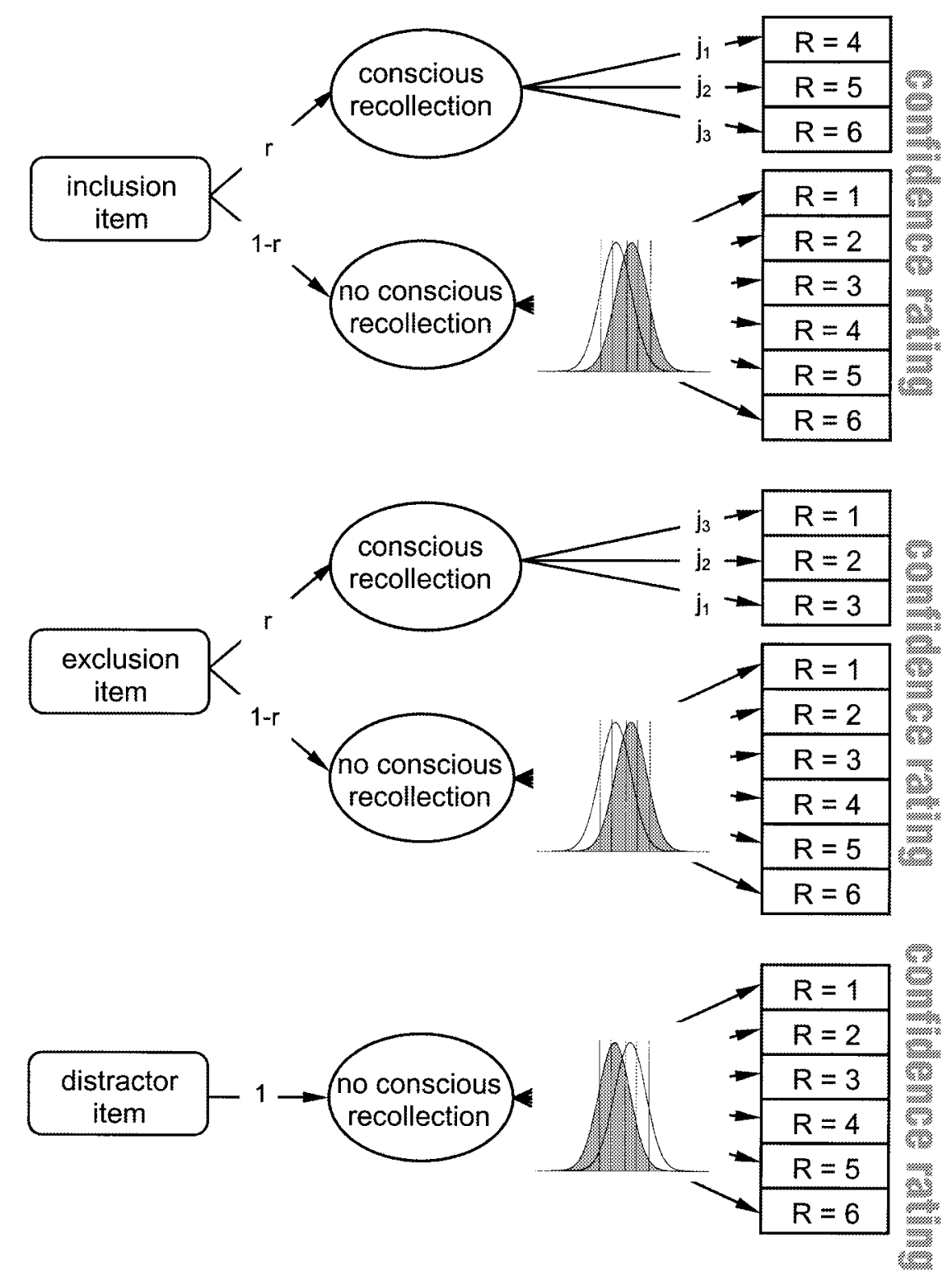

Figure 6. MPTSD. Structure of the model.

function NORMSDIST( ) takes a single argument $z$ and computes the probability $P(Z \leq z)$ that a standard normal random variable $Z$ is smaller or equal to $z$.

The boxes assigned to column $\mathrm{J}$ contain the 18 formulas of the MPTSD(Var). The parameters $r, d^{\prime}, c_{1}, c_{2}, \ldots$, $c_{5}, j_{1}, j_{2}, j_{3}$, and $\sigma$ were named "rstrong V," "dstrong $\mathrm{V}$," "cstrongV1," "cstrongV2," ... " "cstrongV5," "jstrongV1," “jstrongV2," "jstrongV3," and "sigma." The parameter $\sigma$ enters the six equations for modeling the responses of distractor items only (cf. Figure 7C). Note that the square root of the squared standard deviation is used in the model equations. This enables the optimizer to also generate and test negative values of $\sigma$, whereas positive values enter the model equations only.

Figure 8 shows the tables with the model parameters. The upper table (cell range C118:H121) contains the probability parameters $r, j_{1}, j_{2}$, and $j_{3}$. They are computed from the raw parameters $\lambda_{r}, \lambda_{j_{1}}$ and $\lambda_{j_{2}}$ of the lower table (cell range $\mathrm{C} 125: \mathrm{H} 127)$. The parameters in this table are manipulated by the optimizer. The parameters of the MPTSD for simulating the weak and strong items are located in columns D and F, respectively. Column $\mathrm{H}$ contains the parameters of the MPTSD(Var) for simulating the strong items. 
Table 4

MPTSD: The Model Equations

\begin{tabular}{|c|c|c|c|c|}
\hline \multicolumn{5}{|c|}{ Inclusion Item } \\
\hline$P(\mathrm{R}=1)$ & $=$ & & & $(1-r) \cdot \Phi\left(c_{1}-d^{\prime}\right)$ \\
\hline$P(\mathrm{R}=2)$ & $=$ & & & $(1-r) \cdot\left[\Phi\left(c_{2}-d^{\prime}\right)-\Phi\left(c_{1}-d^{\prime}\right)\right]$ \\
\hline$P(\mathrm{R}=3)$ & $=$ & & & $(1-r) \cdot\left[\Phi\left(c_{3}-d^{\prime}\right)-\Phi\left(c_{2}-d^{\prime}\right)\right]$ \\
\hline$P(\mathrm{R}=4)$ & $=$ & $r \cdot j_{1}$ & + & $(1-r) \cdot\left[\Phi\left(c_{4}-d^{\prime}\right)-\Phi\left(c_{3}-d^{\prime}\right)\right]$ \\
\hline$P(\mathrm{R}=5)$ & $=$ & $r \cdot j_{2}$ & & $(1-r) \cdot\left[\Phi\left(c_{5}-d^{\prime}\right)-\Phi\left(c_{4}-d^{\prime}\right)\right]$ \\
\hline$P(\mathrm{R}=6)$ & $=$ & $r \cdot j_{3}$ & & $(1-r) \cdot\left[1-\Phi\left(c_{5}-d^{\prime}\right)\right]$ \\
\hline \multicolumn{5}{|c|}{ Exclusion Item } \\
\hline$P(\mathrm{R}=1)$ & $=$ & $r \cdot j_{3}$ & & $(1-r) \cdot \Phi\left(c_{1}-d^{\prime}\right)$ \\
\hline$P(\mathrm{R}=2)$ & $=$ & $r \cdot j_{2}$ & & $(1-r) \cdot\left[\Phi\left(c_{2}-d^{\prime}\right)-\Phi\left(c_{1}-d^{\prime}\right)\right]$ \\
\hline$P(\mathrm{R}=3)$ & $=$ & $r \cdot j_{1}$ & & $(1-r) \cdot\left[\Phi\left(c_{3}-d^{\prime}\right)-\Phi\left(c_{2}-d^{\prime}\right)\right]$ \\
\hline$P(\mathrm{R}=4)$ & $=$ & & & $(1-r) \cdot\left[\Phi\left(c_{4}-d^{\prime}\right)-\Phi\left(c_{3}-d^{\prime}\right)\right]$ \\
\hline$P(\mathrm{R}=5)$ & $=$ & & & $(1-r) \cdot\left[\Phi\left(c_{5}-d^{\prime}\right)-\Phi\left(c_{4}-d^{\prime}\right)\right]$ \\
\hline$P(\mathrm{R}=6)$ & $=$ & & & $(1-r) \cdot\left[1-\Phi\left(c_{5}-d^{\prime}\right)\right]$ \\
\hline \multicolumn{5}{|c|}{ Distractor Item } \\
\hline$P(\mathrm{R}=1)$ & $=$ & $\Phi\left(c_{1}\right)$ & & \\
\hline$P(\mathrm{R}=2)$ & $=$ & $\Phi\left(c_{2}\right)$ & $-\Phi(c$ & \\
\hline$P(\mathrm{R}=3)$ & $=$ & $\Phi\left(c_{3}\right)$ & $-\Phi(c$ & \\
\hline$P(\mathrm{R}=4)$ & $=$ & $\Phi\left(c_{4}\right)$ & $-\Phi(c$ & \\
\hline$P(\mathrm{R}=5)$ & $=$ & $\Phi\left(c_{5}\right)$ & $-\Phi(c$ & \\
\hline$P(\mathrm{R}=6)$ & $=$ & $1-\Phi$ & & \\
\hline \multicolumn{5}{|c|}{$\begin{array}{l}\text { Note-P(R }=i)=\text { probability of response category } i(1 \leq i \leq 6) ; r= \\
\text { probability of conscious recollection; } j_{k}=\text { probability of choosing re- } \\
\text { sponse category } k+3 \text { for inclusion items and response category } 4-k \\
\text { for exclusion items in case of consciously recollected items }(1 \leq \\
k \leq 3) ; c_{i-}=\text { value of response criterion } i \text { of the signal detection model } \\
\text { (if the familiarity of an item is greater than } c_{i} \text { a response category } \\
\text { greater than category } i \text { is chosen); } d^{\prime}=\text { sensitivity parameter of the sig- } \\
\text { nal detection model; } \Phi()=\text { standard normal distribution function. }\end{array}$} \\
\hline
\end{tabular}

The three formulas, located in the cell range H118:H121, compute the probability parameters of the MPTSD(Var). They implement logistic distributions for mapping raw parameters to probability parameters. The formula in the first box implements the equation

$$
r=\frac{\exp \left(\lambda_{r}\right)}{1+\exp \left(\lambda_{r}\right)} .
$$

$\lambda_{r}$ denotes the raw parameter, and $r$ refers to the probability parameter. The array formula in the second box computes $j_{1}$ and $j_{2}$ from $\lambda_{j_{1}}$ and $\lambda_{j_{2}}$. It implements the equations

$$
j_{1}=\frac{\exp \left(\lambda_{j_{1}}\right)}{1+\sum_{k=1}^{2} \exp \left(\lambda_{j_{k}}\right)} \text {, }
$$

and

$$
j_{2}=\frac{\exp \left(\lambda_{j_{2}}\right)}{1+\sum_{k=1}^{2} \exp \left(\lambda_{j_{k}}\right)} .
$$

The array formula in the last box calculates $j_{3}$ from $\lambda_{j_{1}}$ and $\lambda_{j_{2}}$ :

$$
j_{3}=\frac{1}{1+\sum_{k=1}^{2} \exp \left(\lambda_{j_{k}}\right)} .
$$

Equations 1, 2A, 2B, and 2C enable the optimizer to perform unconstrained optimization on the raw parameters $\lambda_{r}, \lambda_{j_{1}}, \lambda_{j_{2}}$, and $\lambda_{j_{3}}$, whereas the probability parameters $r, j_{1}, j_{2}$, and $j_{3}$ that enter the model equations conform to the constraints imposed on probabilities. For example, Equations $2 \mathrm{~A}, 2 \mathrm{~B}$, and $2 \mathrm{C}$ ensure that $j_{k} \in(0,1)$ $(k=1,2,3)$ and that $j_{1}+j_{2}+j_{3}=1$.

Figure 9 shows the log-likelihood functions and chisquare statistics. The model parameters are estimated by means of maximum likelihood estimation. Cells C139, E139, and G139 contain the kernels of the log-likelihood

Table 5

Multinomial Processing Tree Signal Detection: Symbols and Corresponding Names Used Within the Workbook for Implementing the Models

\begin{tabular}{llll}
\hline \multicolumn{1}{c}{ Symbol } & \multicolumn{1}{c}{ Name } & Address & \multicolumn{1}{c}{ Meaning } \\
\hline$n^{\text {weak }}$ & nweak & B97:B114 & Observed frequencies (MPTSD: weak items) \\
$n^{\text {strong }}$ & nstrong & C97:C114 & Observed frequencies (MPTSD: strong items) \\
$\mu^{\text {weak }}$ & mweak & E97:E114 & Estimated frequencies (MPTSD: weak items) \\
$\mu^{\text {strong }}$ & mstrong & F97:F114 & Estimated frequencies (MPTSD: strong items) \\
$\mu^{\text {strong (Var) }}$ & mstrongV & G97:G114 & Estimated frequencies (MPTSD(Var): strong items) \\
$\pi^{\text {weak }}$ & pweak & H97:H114 & Estimated probabilities (MPTSD: weak items) \\
$\pi^{\text {strong }}$ & pstrong & I97:I114 & Estimated probabilities (MPTSD: long items) \\
$\pi^{\text {strong (Var) }}$ & pstrongV & J97:J114 & Estimated probabilities (MPTSD(Var): strong items) \\
$r^{\text {weak }}$ & rweak & D118:D118 & Parameter $r$ (MPTSD: weak items) \\
$r^{\text {strong }}$ & rstrong & F118:F118 & Parameter $r$ (MPTSD: strong items) \\
$r^{\text {strong (Var) }}$ & rstrongV & H118:H118 & Parameter $r$ (MPTSD(Var): strong items) \\
$j_{1-3}$ weak & jweak1-3 & D119:D121 & Parameters $j_{1}-j_{3}$ (MPTSD: weak items) \\
$j_{1-3}{ }^{\text {strong }}$ & jstrong1-3 & F119:F121 & Parameters $j_{1}-j_{3}$ (MPTSD: strong items) \\
$j_{1-3}{ }^{\text {strong (Var) }}$ & jstrongV1-3 & H119:H121 & Parameters $j_{1}-j_{3}$ (MPTSD(Var): strong items) \\
$d^{\text {weak }}$ & dweak & D128:D128 & Parameter $d^{\prime}$ (MPTSD: weak items) \\
$d^{\text {strong }}$ & dstrong & F128:F128 & Parameter $d^{\prime}$ (MPTSD: strong items) \\
$d^{\text {strong (Var) }}$ & dstrongV & H128:H128 & Parameter $d^{\prime}$ (MPTSD(Var): strong items) \\
$c_{1-5} 5^{\text {weak }}$ & cweak1-5 & D129:D133 & Parameters $c_{1}-c_{5}$ (MPTSD: weak items) \\
$c_{1-5} 5^{\text {strong }}$ & cstrong1-5 & F129:F133 & Parameters $c_{1}-c_{5}$ (MPTSD: strong items) \\
$c_{1-5} 5^{\text {strong (Var) }}$ & cstrongV1-5 & H129:H133 & Parameters $c_{1}-c_{5}$ (MPTSD(Var): unequal variances) \\
$\sigma^{\text {distractor }}$ & sigma & H134:H134 & Standard deviation of the distribution of distractor items \\
\hline
\end{tabular}


A

\begin{tabular}{|c|c|c|c|c|c|c|c|c|c|c|c|}
\hline & A & B & C & D & E & $F$ & \multicolumn{2}{|c|}{ G } & H & 1 & $J$ \\
\hline \multirow{2}{*}{93} & \multicolumn{5}{|c|}{ DATA AND ESTIMATES OF EXPERIMENT 3} & & & & & & \\
\hline & & & & & & & & & & & \\
\hline 94 & Rating & \multirow{2}{*}{\multicolumn{2}{|c|}{ Item Strength }} & & & & & & & & \\
\hline 95 & $1=$ sure new & & & & & & & & & & \\
\hline 96 & $6=$ sure old & weak & strong & & $\mu^{\text {weak }}$ & $\mu^{\text {strong }}$ & $\mu^{\text {stron }}$ & (Var) & $\pi^{\text {weak }}$ & $\pi^{\text {strong }}$ & $\pi^{\text {strong (Var) }}$ \\
\hline 97 & 1 & 194 & 167 & \multirow{6}{*}{$\begin{array}{l}\frac{}{0} \\
\frac{0}{2} \\
\frac{7}{0}\end{array}$} & 182.429 & 136.468 & 163 & 38 & $\rightarrow 0.166$ & 0.120 & $\rightarrow 0.144$ \\
\hline 98 & 2 & 190 & 132 & & 184.234 & 144.830 & 133 & & $\rightarrow 0.167$ & 0.127 & $\rightarrow 0.117$ \\
\hline 99 & 3 & 150 & 123 & & 161.463 & 144.809 & 127 & & $\rightarrow 0.147$ & 0.127 & $\rightarrow 0.112$ \\
\hline 100 & 4 & 135 & 136 & & 144.888 & 138.669 & 139 & & $\rightarrow 0.132$ & 0.122 & $\rightarrow 0.123$ \\
\hline 101 & 5 & 177 & 210 & & 178.006 & 198.642 & 199 & & $\rightarrow 0.162$ & 0.174 & $\rightarrow 0.175$ \\
\hline \multirow[t]{2}{*}{102} & 6 & 254 & 372 & & 248.981 & 376.582 & 374 & & $\rightarrow 0.226$ & 0.330 & $\rightarrow 0.329$ \\
\hline & & & & & $\uparrow$ & 1 & 1 & & & & \\
\hline \multicolumn{3}{|c|}{$=\mathrm{SUM}(\mathrm{B} 97: \mathrm{B} 102)^{\star} \mathrm{H} 97: \mathrm{H} 102$} & \multicolumn{5}{|c|}{$=(1 \text { rweak })^{*}$ NORMSDIST (cweak 1-dweak) } & \multicolumn{4}{|c|}{$=(\hat{1} \text {-rstrongV })^{\star} \mathrm{NORMSDIST}$ (cstrongV1-dstrongV) } \\
\hline \multicolumn{3}{|c|}{$=$ SUM(C97:C102)*197:1102\} } & \multicolumn{5}{|c|}{$\begin{array}{l}=(1-\text { rweak })^{*}(\text { NORMSDIST(cweak2-dweak)- } \\
\text { NORMSDIST(cweak1-dweak)) }\end{array}$} & \multicolumn{4}{|c|}{$\begin{array}{l}\text { =(1-rstrongV)*(NORMSDIST(cstrongV2-dstrongV)- } \\
\text { NORMSDiST(cstrongV1-dstrongV)) }\end{array}$} \\
\hline in & 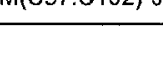 & 01023 & \multicolumn{5}{|c|}{$\begin{array}{l}=\left(1-\text { rweak) }{ }^{\star} \text { (NORMSDIST(cweak3-dweak)- }\right. \\
\text { NORMSDIST(cweak2-dweak)) }\end{array}$} & \multicolumn{4}{|c|}{$\begin{array}{l}=(1-\text { rstrongV) })^{*} \text { (NORMSDIST(cstrongV3-dstrongV)- } \\
\text { NORMSDIST(cstrongV2-dstrongV)) }\end{array}$} \\
\hline & & & \multicolumn{5}{|c|}{$\begin{array}{l}\text { =rweak*jweak1 + } \\
\left(1-\text { rweak) }{ }^{*}(\text { NORMSDIST(cweak4-dweak)- }\right. \\
\text { NORMSDIST(cweak3-dweak)) }\end{array}$} & \multicolumn{4}{|c|}{$\begin{array}{l}\text { =rstrongV*jstrongV1 + } \\
(1-\text { rstrongV)*(NORMSDIST(cstrongV4-dstrongV)- } \\
\text { NORMSDiST (cstrongV3-dstrongV)) }\end{array}$} \\
\hline & & & \multicolumn{5}{|c|}{$\begin{array}{l}=r w e a k^{*}{ }^{*} \text { weak2 }+ \\
(1-\text { rweak) } \\
\text { NORMSDIST(cweak4-dweak)) }\end{array}$} & \multicolumn{4}{|c|}{ 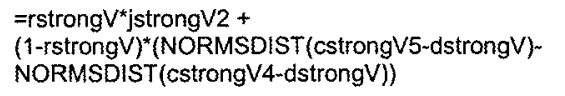 } \\
\hline & & & \multicolumn{5}{|c|}{$\begin{array}{l}\text { =rweak*jweak3 + } \\
(1-\text { rweak })^{*}(1-\text { NORMSDIST (cweak5-dweak)) }\end{array}$} & \multicolumn{4}{|c|}{$\begin{array}{l}\text { "rstrongV*jstrongV3 }{ }^{*} \\
(1-\text { rstrongV) })^{*}(1-N O R M S D I S T \text { (cstrongV5-dstrongV)) }\end{array}$} \\
\hline
\end{tabular}

B

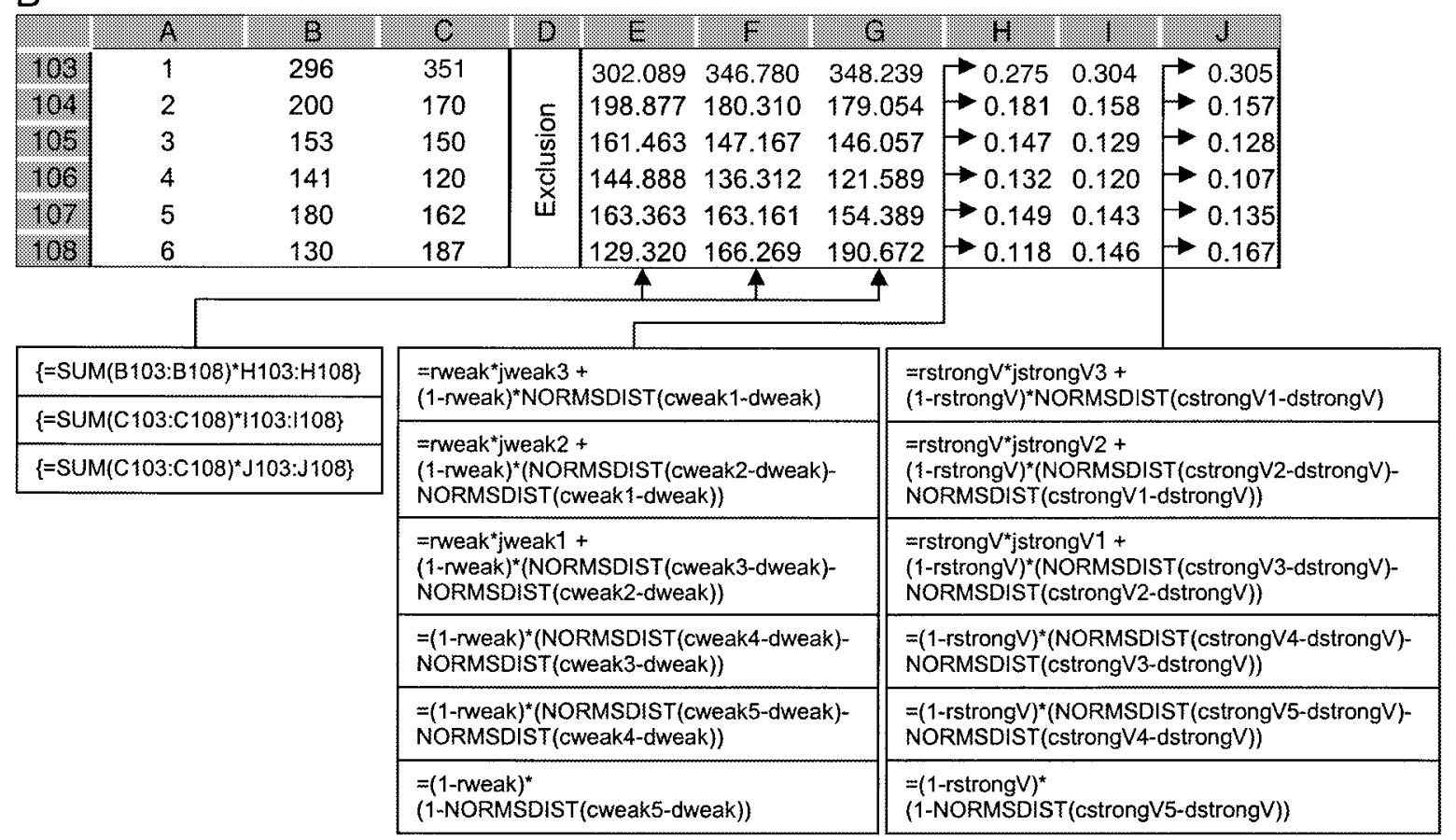

Figure 7. (A) MPTSD. Extract of the worksheet, "Part 1: Data and Model Estimates for Inclusion Items." (B) MPTSD. Extract of the worksheet, "Part 2: Data and Model Estimates for Exclusion Items." 


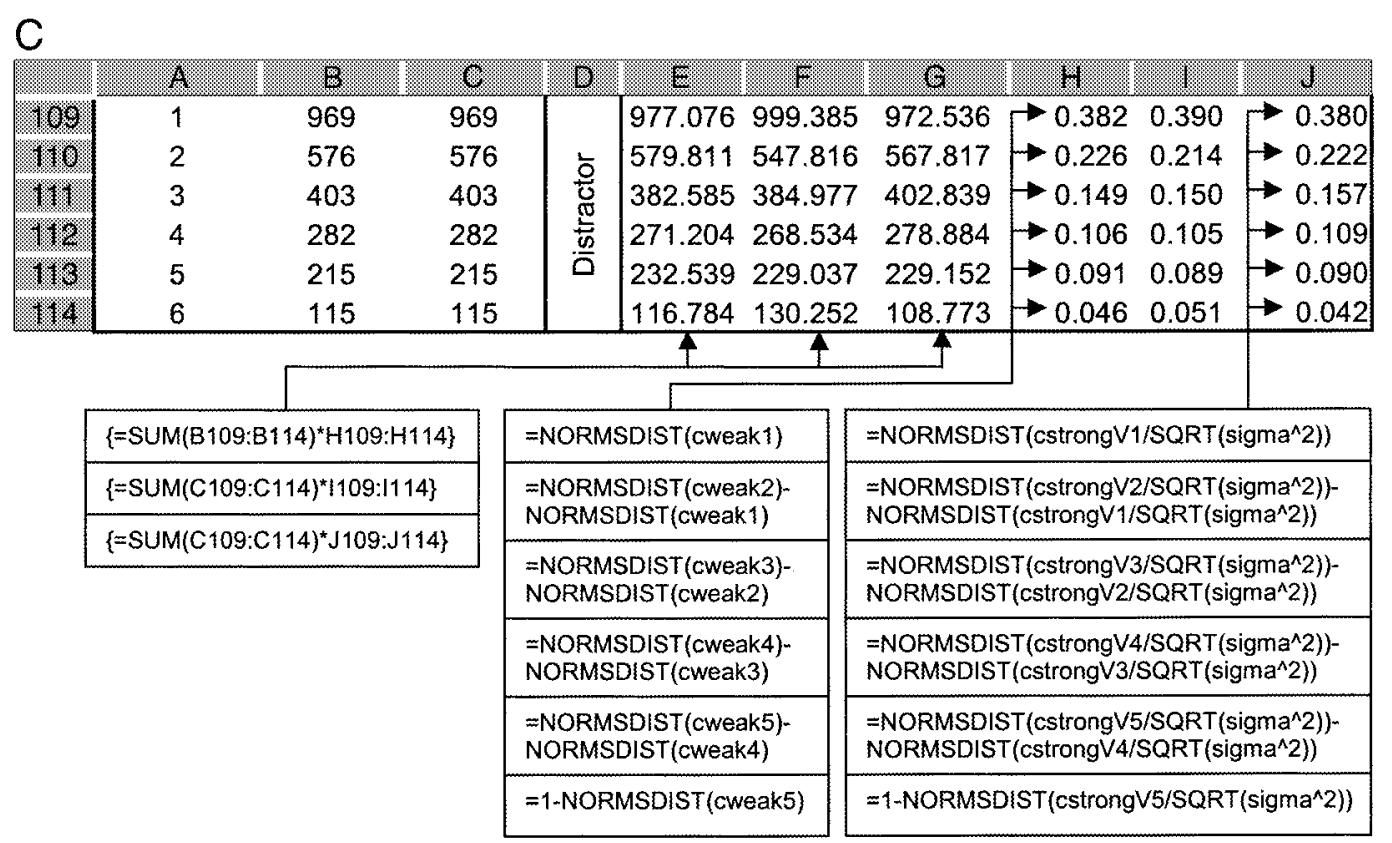

Figure 7 (continued). (C) MPTSD. Extract of the worksheet, Part 3: Data and Model Estimates for Distractor Items.

functions (i.e., the log-likelihood functions without irrelevant constants) of the MPTSD for simulating the weak and strong items and of the MPTSD(Var) for modeling the strong items, respectively. The shown values are the maxima of the log-likelihood functions. The table in cell range A143:F146 contains the Pearson chi-square statistic, the likelihood ratio chi-square statistic, the degrees of freedom, and the probabilities corresponding to the test statistics.

The formula in the box assigned to column B computes the Pearson test statistic $X^{2}$ :

$$
X^{2}=\sum_{\text {cells }} \frac{\left(n_{i}-\hat{\mu}_{i}\right)^{2}}{\hat{\mu}_{i}},
$$

where $n_{i}$ denotes the observed frequency in cell $i(i=1$, $2, \ldots, 18)$, and $\hat{\mu}_{i}$ refers to the predicted frequency. The formula in the box assigned to column $\mathrm{C}$ implements the equation of the likelihood ratio test statistic $G^{2}$ :

$$
G^{2}=2 \cdot \sum_{\text {cells }} n_{i} \cdot \log \left(\frac{n_{i}}{\hat{\mu}_{i}}\right),
$$

where $\log ()$ denotes the natural $\log$ arithm. The corresponding worksheet function is $\mathrm{LN}($ ). The array formulas for computing these statistics generate vectors with the 18 summands and add them together by means of the SUM( ) function.

The worksheet function CHIDIST( ) in the boxes assigned to cells E146 and F146 takes as input the value of the test statistic ( $X^{2}$ and $G^{2}$, respectively), as well as the degrees of freedom, and computes the tail probability of the chi-square distribution (i.e., the probability that a chisquare random variable with the specified degrees of freedom is greater than the test statistic).

The box assigned to cell G139 computes the kernel of the (product multinomial) log-likelihood function for the MPTSD(Var):

$$
\log L=\sum_{\text {cells }} n_{i} \cdot \log \hat{\pi}_{i}
$$

where $\log L$ denotes the $\log$-likelihood, and $\hat{\pi}_{i}$ refers to the predicted probability. The shown formula contains an IF( ) expression for testing whether the predicted probability is smaller or equal to zero. In this case, the function adds the value $-1,000$ to the likelihood instead of computing the undefined logarithm. This prevents the optimizer from producing undefined solutions with nonpositive predicted probabilities. The shown formula is an array function that generates a vector with 18 summands and adds them together by means of the SUM() function.

Having implemented the models and likelihood functions, the built-in optimizer, the Solver (menu command Tools/Solver), can be used for estimating parameters (the Solver is not available by default; to install it, use the menu command Tools/Add-Ins and check the Solver Add-In checkbox). The menu for configuring the Solver requires at least two specifications: (1) the cell with the to be optimized function (e.g., cell G139), and (2) the cells with the model parameters (e.g., cell range H125:H134). (The menu allows for additional specifications. In the present case, the following configuration was used: optimization method, Newton method with central derivates; precision, 1E-16; tolerance, 0 ; and convergence, 1E-16.) 


\begin{tabular}{|c|c|c|c|c|c|c|c|c|}
\hline & 1 & 8. & 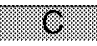 & (1) & e. & F & (4. & भit: \\
\hline \multicolumn{9}{|c|}{116 ESTIMATED PROBABILITY PARAMETERS } \\
\hline \multicolumn{9}{|c|}{ 117. $\quad\left(\begin{array}{l|l|l|} & & \\
\end{array}\right.$} \\
\hline 118 & & & $r^{\text {weak }}$ & 0.122 & $r^{\text {strong }}$ & 0.218 & $r^{\text {strong(Var) }}$ & 0.218 \\
\hline 119 & & & $\mathrm{j}_{1}$ weak & 0.000 & $j_{1}^{\text {strong }}$ & 0.010 & $\mathrm{j}_{1}^{\text {strong (Var) }}$ & 0.073 \\
\hline 120. & & & $\mathrm{j}_{2}{ }^{\text {weak }}$ & 0.109 & $\mathrm{j}_{2}$ strong & 0.143 & $\mathrm{j}_{2}$ strong (Var) & 0.183 \\
\hline 121 & & & $j_{3}^{\text {weak }}$ & 0.891 & $\mathrm{j}_{3}^{\text {strong }}$ & 0.848 & $\mathrm{j}_{3}$ strong (Var) & 0.743 \\
\hline \multicolumn{9}{|l|}{122} \\
\hline \multicolumn{9}{|c|}{ 128. ESTIMATED PARAMETERS } \\
\hline \multicolumn{9}{|c|}{\begin{tabular}{l|l|l|l|} 
& \\
\end{tabular}} \\
\hline 125 & & 1 & $\lambda_{r}^{\text {weak }}$ & -1.973 & $\lambda_{r}^{\text {strong }}$ & -1.279 & $\lambda_{r}^{\text {strong (Var) }}$ & -1.280 \\
\hline 126 & & 2 & $\lambda_{\mathrm{j} 1}{ }^{\text {weak }}$ & -21.134 & $\lambda_{\mathrm{j} 1}^{\text {strong }}$ & -4.491 & $\lambda_{j 1}{ }^{\text {strong (Var) }}$ & -2.316 \\
\hline 127 & & 3 & $\lambda_{\mathrm{j} 2}{ }^{\text {weak }}$ & -2.101 & $\lambda_{j 2}{ }^{\text {strong }}$ & -1.780 & $\lambda_{j 2}{ }^{\text {strong (Var) }}$ & -1.399 \\
\hline 128 & & 4 & $d^{\text {weak }}$ & 0.581 & $d^{\text {strong }}$ & 0.745 & $d^{\text {strong (Var) }}$ & 0.646 \\
\hline 129 & & 5 & $c_{1}{ }^{\text {weak }}$ & -0.301 & $\mathrm{c}_{1}^{\text {strong }}$ & -0.278 & $\mathrm{C}_{1}^{\text {strong (Var) }}$ & -0.255 \\
\hline 130 & & 6 & $\mathrm{c}_{2}^{\text {weak }}$ & 0.275 & $\mathrm{C}_{2}^{\text {strong }}$ & 0.265 & $\mathrm{c}_{2}^{\text {strong (Var) }}$ & 0.215 \\
\hline 131: & & 7 & $\mathrm{c}_{3}$ weak & 0.699 & $\mathrm{C}_{3}$ strong & 0.690 & $\mathrm{C}_{3}{ }^{\text {strong }(\text { Var) }}$ & 0.588 \\
\hline 182 & & 8 & $\mathrm{C}_{4}$ weak & 1.096 & $\mathrm{C}_{4}^{\text {strong }}$ & 1.079 & $\mathrm{C}_{4}$ strong (Var) & 0.933 \\
\hline 180 & & 9 & $\mathrm{C}_{5}$ weak & 1.689 & $\mathrm{C}_{5}^{\text {strong }}$ & 1.636 & $\mathrm{C}_{5}$ strong (Var) & 1.439 \\
\hline \multirow[t]{4}{*}{184} & & 10 & & & & & $\sigma^{\text {distractor }}$ & 0.835 \\
\hline & & & & \multicolumn{5}{|c|}{$=E X P(H 125) /(1+E X P(H 125))$} \\
\hline & & & & \multicolumn{5}{|c|}{$=\operatorname{EXP}(H \$ 126: H \$ 127) /(1+\operatorname{SUM}(\operatorname{EXP}(H \$ 126: H \$ 127)))$} \\
\hline & & & & \multicolumn{5}{|c|}{$=1 /(1+S U M(E X P(H \$ 126: H \$ 127)))$} \\
\hline
\end{tabular}

Figure 8. MPTSD. Extract of the worksheet, Part 4: Tables with Model Parameters.

\section{Results of the Simulation}

Table 6 presents the fit statistics of different models for the three experiments of Yonelinas (1994). The MPTSD does not fit the short lists in Experiment 2 and the strong items in Experiment 3. The MPTSD(Var) fits these data (due to the high number of observations, I assume a critical $p$ value of .001). The results justify the conclusion that, contrary to the claim of Yonelinas (1994), the process of conscious recollection cannot account for the unequal variances of the distribution of new and old items.

\section{DISCUSSION}

In the following, I first summarize the main components of the implementation. Then, I discuss the pros and cons of spreadsheets versus standard statistical packages for building statistical models.

\section{Organization of the Model on the Spreadsheet}

The implementation of the model is made up of five components: (1) the table with raw parameters (cf. Figure 8 , lower table) that are manipulated by the Solver, (2) the table with probability parameters (cf. Figure 8, upper table), (3) the 18 model equations (cf. Figures 7A, 7B, and 7C), (4) the log-likelihood function (cf. Figure 9), and (5) the table with the test statistics (cf. Figure 9).
The Solver requires the specification of at least two elements: (1) The cell with the function to be optimized, and (2) the cell range containing the parameters. The likelihood function can be maximized by manipulating parameters due to the following chain of dependencies: parameters $\rightarrow$ model equations $\rightarrow$ probability distributions $\rightarrow$ likelihood function.

\section{Spreadsheets Versus Statistical Packages for Implementing Statistical Models}

Standard statistical packages, such as SPSS or SYSTAT, also provide facilities for building statistical models: statistical functions and an optimizer. These packages have two advantages over spreadsheets. (1) For some log-likelihood functions, they provide a better approximation of the optimum. For example, in maximizing the product Poisson likelihood function, the Solver sometimes produces slightly inferior results than the nonlinear module of SYSTAT. However, the estimated parameters are usually identical up to, at least, five decimal places. (2) The standard packages provide additional statistics (e.g., the asymptotic covariance matrix) that may be used for model diagnosis (e.g., empirical identification of parameters) and for computing important statistics (e.g., confidence intervals).

Unfortunately, the class of multiequation models that can be implemented by means of the nonlinear(regression) 


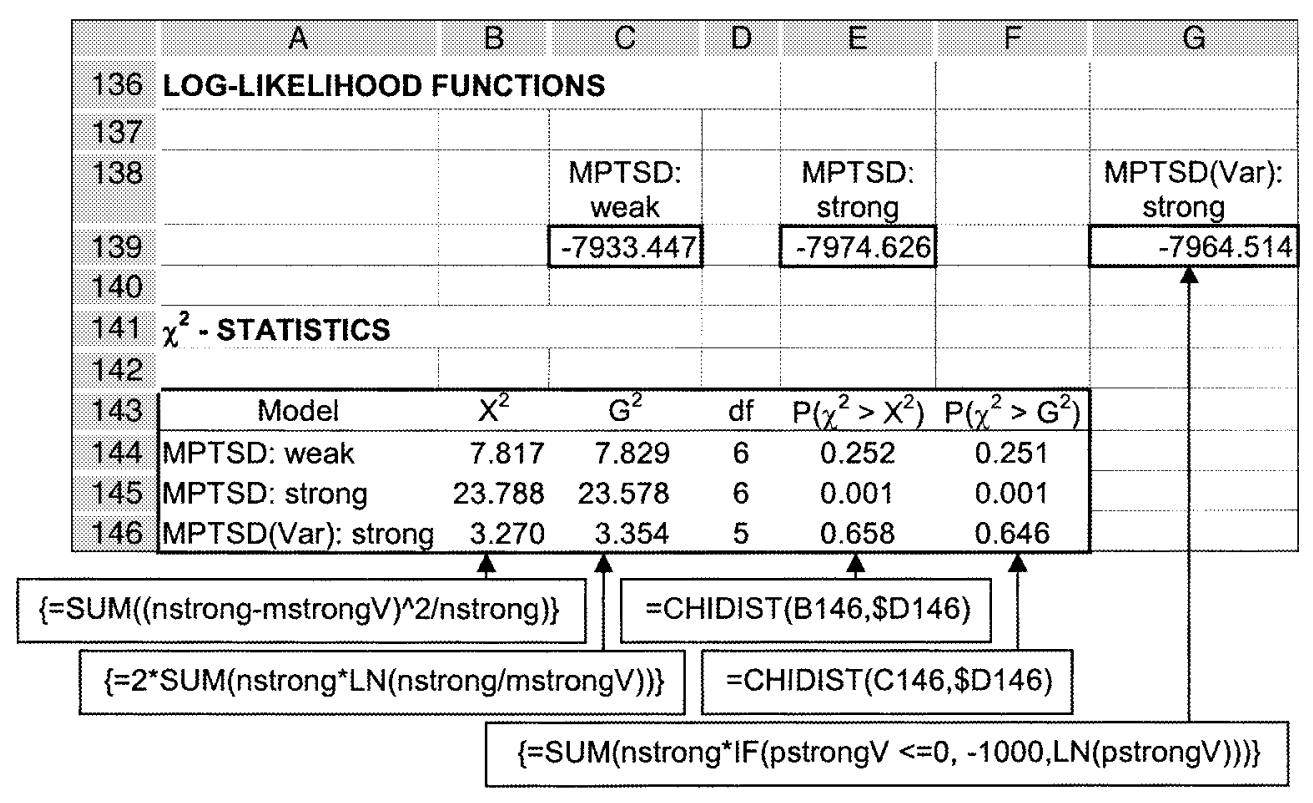

Figure 9. MPTSD. Extract of the worksheet, Part 5: Likelihood Functions and Test Statistics.

modules of the standard statistical packages is limited to general linear models because the whole system of equations must be represented by a single formula. Differences between equations can be modeled by using a design matrix. By contrast, spreadsheets enable the individualdesign of the model equations. Although a pure signal detection model is contained in the class of general linear models (DeCarlo, 1998), the MPTSD is not. Thus, it cannot be implemented by means of the standard statistical packages.

Table 6

Multinomial Processing Tree Signal Detection: Results of Fitting the MPTSD and the MPTSD(Var) to the Data of Yonelinas (1994)

\begin{tabular}{lccc}
\hline \multicolumn{1}{c}{ Model } & $G^{2}$ & $d f$ & $p$ \\
\hline & Experiment 1 & & \\
MPTSD: short & 9.546 & 6 & .145 \\
MPTSD: long & 10.699 & 6 & .098 \\
& Experiment 2 & & \\
MPTSD: short & 26.538 & 6 & .000 \\
MPTSD: long & 17.923 & 6 & .006 \\
MPTSD(Var): short & 12.459 & 5 & .029 \\
& Experiment 3 & & \\
MPTSD: weak & 7.829 & 6 & .251 \\
MPTSD: strong & 23.578 & 6 & .001 \\
MPTSD(Var): strong & 3.354 & 5 & .646 \\
\hline
\end{tabular}

Note-MPTSD = multinomial processing tree signal detection model assuming equal variance of the distributions for old and new items; MPTSD $($ Var $)=$ multinomial processing tree signal detection model, assuming unequal variance of the distributions for old and new items; short $=$ short study lists $($ Experiment 1,10 items per list; Experiment 2, 6 items per list); long = long study lists (Experiment 1, 30 items per list, Experiment 2, 18 items per list); weak = weak items (study time, $1 \mathrm{sec}$ per item); strong = strong items (study time, $3 \mathrm{sec}$ per item).
In summary, standard statistical packages are preferable to spreadsheets for implementing general linear models. Spreadsheets enable the implementation of a broader class of statistical models, however.

\section{Conclusion}

Five major characteristics of spreadsheets are important for implementing cognitive models. (1) The spreadsheet data structure is intuitively reasonable. Its utility is enhanced by additional capabilities, such as the possibility to define names, automatic adjustment of cell references, automatic recomputation in case of modification, and graphical tools that facilitate debugging. (2) Array functions and the associated expansion mechanism enable the implementation of complex computations by means of single expressions. (3) The library of statistical and engineering functions enables the implementation of sophisticated numeric models. (4) The built-in optimizer allows one to estimate model parameters. (5) The graphical capabilities make it possible to generate figures that are automatically adjusted if the underlying data are modified.

These features make spreadsheets a versatile tool for, at least, three groups of people: (1) Researchers with no programming knowledge may implement their models on spreadsheets; (2) instructors may employ spreadsheets for teaching cognitive models; and (3) programmers may find spreadsheets helpful for developing prototypes of models and for testing programs.

\section{REFERENCES}

Baker, A. G., Vallée-Tourangeau, F., \& Murphy, R. A. (2000). Asymptotic judgment of cause in a relative validity paradigm. Memory \& Cognition, 28, 466-479. 
Batchelder, W. H., \& Riefer, D. M. (1990). Multinomial models of source monitoring. Psychological Review, 97, 548-564.

Bayen, U. J., Murnane, K., \& Erdfelder, E. (1996). Source discrimination, item detection, and multinomial models of source monitoring. Journal of Experimental Psychology: Learning, Memory, \& Cognition, 22, 197-215.

Bayen, U. J., Nakamura, G. V., Dupuis, S. E., \& Yang, C.-L. (2000). The use of schematic knowledge about sources in source monitoring. Memory \& Cognition, 28, 480-500.

Buchner, A., Erdfelder, E., \& VAterrodt-Plünnecke, B. (1995). Toward unbiased measurement of conscious and unconscious memory processes within the dissociation framework. Journal of Experimental Psychology: General, 124, 137-160.

DeCarlo, L. T. (1998). Signal detection theory and generalized linear models. Psychological Methods, 3, 186-205.

ERDFELDER,E., \& BUCHNER, A (1998a). Decomposing the hindsight bias: A multinomial processing tree model for separating recollection and reconstruction in hindsight bias. Journal of Experimental Psychology: Learning, Memory, \& Cognition, 24, 387-414.

ERdFELDER, E., \& BUCHNER, A (1998b). Process-dissociation measurement models: Threshold theory or detection theory? Journal of Experimental Psychology: General, 127, 83-96.

Hu, X., \& BATCHELDER, W. H. (1994). The statistical analysis of general processing tree models with the EM algorithm. Psychometrica, 59, 21-47.

JACOBY, L. L. (1991). A process dissociation framework: Separating automatic from intentional uses of memory. Journal of Language \& Memory, 30, 513-541.

KLAUER, K. C., \& WEGENER, I. (1998). Unraveling social categorization in the "Who said what?" paradigm. Journal of Personality \& Social Psychology, 75, 1155-1178.

KRUSCHKE, J. K. (1992). ALCOVE: An exemplar-based connectionist model of category learning. Psychological Review, 99, 22-44.

Масно, S. (in press). Kognitive Modellierung mit neuronalen Netzen: Eine praktisch orientierte Einführung [Cognitive modeling with neural networks: An applied introduction] (Freiburger Beiträge zur Psychologie). Bern: Hans Huber.

Macmillan, N. A., \& Creelman,D. C. (1991).Detection theory: A user's guide. Cambridge: Cambridge University Press.

Orvis, W. J. (1996). Excel for scientists and engineers. San Francisco: Sybex.

Pearce, J. M. (1987). A model for stimulus generalization in Pavlovian conditioning. Psychological Review, 94, 61-73.

Pearce, J. M. (1994). Similarity and discrimination: A selective review and a connectionist model. Psychological Review, 101, 587-607.
RatclifF, R, Sheu, C.-F., \& Gronlund, S. D. (1992). Testing global memory models using ROC curves. Psychological Review, 99, 518-535.

Rescorla, R. A., \& Wagner, A. R. (1972). A theory in Pavlovian conditioning: Variations in the effectiveness of reinforcement and nonreinforcement. In A. H. Blach \& W. F. Prokasy (Eds.), Classical conditioning II: Current research and theory (pp. 64-99). New York: Appleton-Century-Crofts.

RIEFER, D. M., \& BATCHELDER, W. H. (1988). Multinomial modeling and the measurement of cognitive processes. Psychological Review, 95, 318-339.

Shanks, D. R., Charles, D., Darby, R. J., \& Azmi, A. (1998). Configural processes in human associative learning. Journal of Experimental Psychology: Learning, Memory, \& Cognition, 24, 1353-1378.

Vallée-Tourangeau,F., Murphy, R. A., \& Baker, A. G. (1998).Causal induction in the presence of a perfect negative cue: Contrasting predictions from associative and statistical models. Quarterly Journal of Experimental Psychology, 51B, 173-191.

Vallée-Tourangeau, F., Murphy, R. A., Drew, S., \& Baker, A. G. (1998). Judging the importance of constant and variable candidate causes: A test of the Power PC theory. Quarterly Journal of Experimental Psychology, 51A, 65-84.

Van Overwalle, F., \& Rooy, D. F. (1998). A connectionist approach to causal attribution. In S. J. Read \& L. C. Miller (Eds.), Connectionistmodels of social reasoning and social behavior. (pp. 143-171). Mahwah, NJ: Erlbaum.

YonelinAs, A. P. (1994). Receiver-operating characteristics in recognition memory: Evidence for a dual-process model. Journal of Experimental Psychology: Learning, Memory, \& Cognition, 20, 1341-1354.

Yonelinas, A. P., \& JACOBY, L. L. (1996). Response bias and the processdissociation procedure. Journal of Experimental Psychology: General, 125, 422-434.

\section{NOTE}

1. Let $\mathbf{v}$ denote an arbitrary vector with $I$ components. $v$ is normalized by dividing each component by the length $\|v\|$ of $v$. The (Euclidean) length of a vector is the square root of the scalar product:

$$
\|\boldsymbol{v}\|=\sqrt{\boldsymbol{v}^{\mathrm{T}} \cdot \boldsymbol{v}}=\sqrt{\sum_{i=1}^{I} v_{i}^{2}} .
$$

(Manuscript received October 4, 2000; revision accepted for publication August 5, 2001). 